\title{
A systematic data-driven Demand Side Management method for smart natural gas supply systems
}

Huai Su ${ }^{\text {a }}$, Enrico Zio b,c , Jinjun Zhang a ${ }^{\text {a }}$, Lixun Chi ${ }^{\text {a }}$, Xueyi Li ${ }^{\text {a }}$, Zongjie Zhang a, d

${ }^{a}$ National Engineering Laboratory for Pipeline Safety/ MOE Key Laboratory of Petroleum Engineering /Beijing Key Laboratory of Urban Oil and Gas Distribution Technology, China University of Petroleum-Beijing, 102249, Beijing, China

${ }^{\mathrm{b}}$ Dipartimento di Energia, Politecnico di Milano, Via La Masa 34, 20156, Milano,

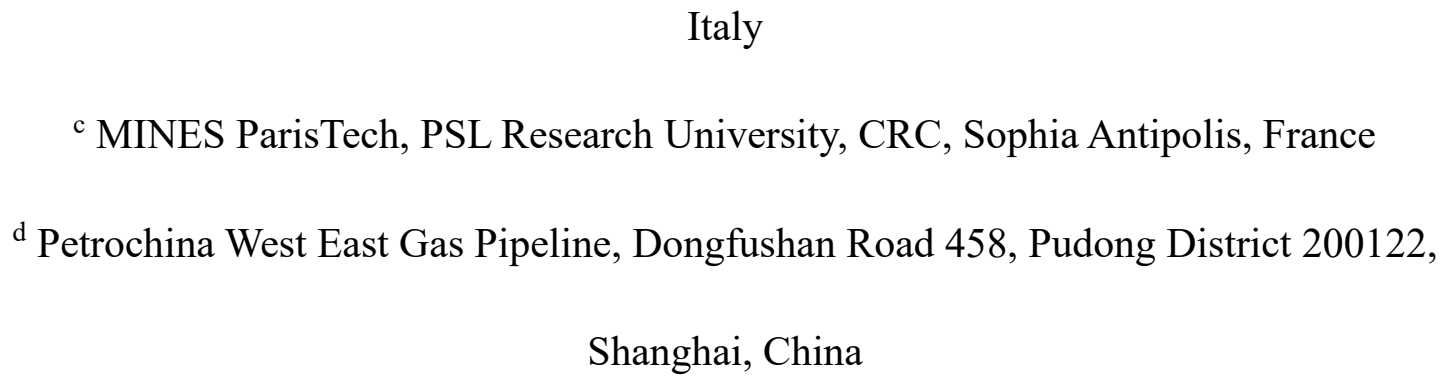

\begin{abstract}
Advanced sensor and communication technologies can make natural gas supply systems smarter than ever before, in both system management and operation. This paper presents the development of a novel data-driven Demand Side Management, whose framework includes demand forecasting, customer response analysis, prediction of dynamic condition of the gas network, quick supply reliability evaluation, multi-objective optimization and decision-making. The aims of this DSM method are to smooth load profiles, improve company profit and enhance system reliability, by means of a dynamic pricing strategy. To verify the effectiveness of the developed framework, a
\end{abstract}

\footnotetext{
${ }^{*}$ Corresponding author. Address: College of Mechanical and Transportation Engineering, China University of Petroleum, Fuxue Road 18, Changping District 102249, Beijing, China. Tel.: +86-10-8973 4627; fax: +86-10-8973 4627. E-mail address: zhangjj@cup.edu.cn
} 
case study is considered, concerning the management of a relatively complex gas supply system, wherein four different pricing periods are introduced for comprehensively testing. The results in the case study show that the DSM framework is able to effectively achieve the targets of peak shaving and valley filling. Besides, it can significantly and stably improve the system efficiency and reliability, for different pricing periods. Finally, pricing period determination is discussed in relation to the features of performance.

Keywords: Natural gas supply system; Demand Side Management; Data-driven; Deep learning;

Multi-objective optimization

\begin{tabular}{|c|c|c|c|}
\hline \multicolumn{4}{|c|}{ Nomenclature } \\
\hline CPP & Critical Peak Pricing & $R R$ & risk reduction \\
\hline DR & Demand Response & $S R$ & risk of natural gas shortage \\
\hline $\mathrm{DCM}$ & Demand Side & Supply & the maximal total supply \\
\hline DSIN & Management & Capacity $_{\text {total }, \max }$ & $\begin{array}{l}\text { capacity } \\
\text { customer utility for }\end{array}$ \\
\hline LNG & liquified natural gas & $U c$ & $\begin{array}{l}\text { consequence quantification of } \\
\text { risk }\end{array}$ \\
\hline LSTM & $\begin{array}{l}\text { Long Short Term } \\
\text { Memory }\end{array}$ & Us & profit of supplier \\
\hline MAE & Mean Absolute Error & $U_{i}$ & utility of the Customer $i$ \\
\hline MRE & Mean Relative Error & $c$ & speed of sound \\
\hline RTP & Real-Time Pricing & $c s$ & silhouette width \\
\hline RNN & recurrent neural network & cost & $\begin{array}{l}\text { total cost of gas production and } \\
\text { transportation }\end{array}$ \\
\hline RMSE & Root Mean Squared Error & $d$ & forecasted demand \\
\hline TOU & Time Of Use & $l$ & customer consumption \\
\hline UGS & underground gas storage & $p r$ & natural gas price at time \\
\hline$A$ & cross-section area & $\begin{array}{l}p_{i} \\
p_{i, \text { prediction }}\end{array}$ & $\begin{array}{l}\text { delivery pressure at node } i \\
\text { predicted delivery pressure at } \\
\text { customer } i\end{array}$ \\
\hline$A C F R$ & $\begin{array}{l}\text { aggregate consumption } \\
\text { fluctuation reduction }\end{array}$ & $q$ & squash factor \\
\hline$A C S$ & $\begin{array}{l}\text { aggregate customers' } \\
\text { satisfaction }\end{array}$ & $r_{c}$ & cluster radius \\
\hline$C S$ & cluster silhouette & $s$ & customer satisfaction \\
\hline E & demand elasticity of price & $t$ & time $t$ \\
\hline
\end{tabular}




\begin{tabular}{|c|c|c|c|}
\hline$F_{\text {optimal }}^{\mathrm{s}}$ & $\begin{array}{l}\text { representative solution in } \\
\text { family } s\end{array}$ & $v_{i, t}, k_{i, t}$ & positive constant factor \\
\hline$G$ & objective value & $\Phi$ & $\begin{array}{l}\text { cumulative standard Gaussian } \\
\text { distribution }\end{array}$ \\
\hline$K$ & $\begin{array}{l}\text { the number of clustered } \\
\text { families }\end{array}$ & $\psi_{\text {accept }}, \psi_{\text {reject }}$ & accept ratio and reject ratio \\
\hline$L$ & gas consumptiom & $\beta_{\text {demand }}$ & $\begin{array}{l}\text { reliability coefficient of } \\
\text { customer }\end{array}$ \\
\hline$L F$ & load fluctuation & $\mu, \sigma$ & $\begin{array}{l}\text { mean value and variances of } \\
\text { relevant parameters }\end{array}$ \\
\hline$L_{\text {total }}$ & $\begin{array}{l}\text { total natural gas } \\
\text { consumption }\end{array}$ & $\tau_{M}$ & \\
\hline$P I$ & profit improvement & $\Delta t$ & $\begin{array}{l}\text { average duration of time that } \\
\text { the consumption of the line- } \\
\text { pack capacity }\end{array}$ \\
\hline$P R$ & peak reduction & $\Delta x_{i, j}$ & $\begin{array}{l}\text { length of the part of Pipeline } j \\
\text { connection customer } i\end{array}$ \\
\hline$P\left(G_{\text {norm }}(\gamma)\right)$ & $\begin{array}{l}\text { potential of } G_{\text {norm }}(\gamma) \\
\text { the customer contracted }\end{array}$ & $\rho$ & gas density \\
\hline$P_{\text {lim }, i}$ & $\begin{array}{l}\text { minimum delivery } \\
\text { pressure }\end{array}$ & $\gamma$ & vector of objective values \\
\hline$Q_{i, j}$ & $\begin{array}{l}\text { gas flow into delivery } \\
\text { node } i \text { from the } \\
\text { connecting pipeline } j\end{array}$ & & \\
\hline
\end{tabular}

\section{Introduction}

Natural gas is an important energy resource because of its natural benefit including relatively reduced capital cost, high energy containing value, low greenhouse gas emission and so on. Besides, that, its stability of production and supply makes it more competitive than other clean energy resources, like wind power and solar energy, in an integrated energy system [1]. According to the estimation of the International Energy Agency, by 2030, 25\% of the world energy consumption will be covered by natural gas [2]. Hence, it is an essential issue to maintain a supply-demand balance of natural gas. 
to different customers, e.g., large local distribution networks, gas-fired power plants, large industries, etc. Such natural gas supply networks are now always closely integrated into the integrated energy systems, as an important component for flexibility improvement, peak-shaving and coping with the uncertainties of some renewable energy sources. Considering these issues, supply reliability and operation efficiency of the natural gas transmission pipeline networks becomes even more fundamental than before, for energy supply security and its efficiency.

The reliability of natural gas supply systems is threatened by a number of problems, such as uncertain operation conditions, complex environments, rapidly increasing natural gas market demands and significant demand fluctuations from the customers. In particular, short-term demand fluctuation is a difficult problem, which decreases gas supply reliability, system efficiency and production profit. The main reason for these short-term fluctuations is that the gas price is kept as fixed on relatively long time horizons (several seasons or years) and the customers, unaffected by the strains on the gas supply systems, use gas just as their habits, which can cause high peaks during the day and low valleys in the night.

To deal with this problem, smart grids and smart energy systems are developing advanced Demand Side Management (DSM) strategies to reduce the peaks and fill the valleys by influencing customers' consumption patterns, via economic methods [3]-[5]. These DSM strategies can be classified into different groups according to different principles. In this paper, the DSMs are mainly categorized as energy efficiency methods and Demand Response (DR) methods [6], according to the timing and impact of the DSM methods on the customers. Energy efficiency methods include all the permanent efficiency improvement methods [7], for example, equipment replacement and system update. DR methods can control the patterns of the customers' load [8]-[10]. Furtherly, 
according to the way to influence the customers, the DR methods can be classified as Incentivebased DR and Time-based DR. The Incentive-based DR are triggered by specific situations [6], [11]:

for example, special contracts for some specific customers with limited sheds, voluntary behaviors to emergency signals [12], customers bidding for curtailing at reasonable prices, etc. The Timebased DR methods are mainly performed by changing the price of gas in time to desired demand. According to the rules to adjust the price, the Time-based DR methods can be grouped by Time-ofUse (TOU) method [13], Critical Peak Pricing (CPP) method [14] and Real-Time Pricing (RTP) method [15]. From the literature review, it emerges that the Time-based DR methods are the most effective DSM strategies, because their inherent characteristics are more suitable to the real-world unsteady and fluctuating energy consumptions. This paper focuses on developing a DSM strategy from the Time-based perspective. Properties of the three types of Time-based methods and survey results are presented in Table 1.

Table 1 Survey about the different kinds of Time-based DR methods

\begin{tabular}{c|c|c}
\hline & $\begin{array}{c}\text { Main } \\
\text { characteristics }\end{array}$ & Applications \\
\hline Time-of-Use & $\begin{array}{c}\text { Static price } \\
\text { schedule }\end{array}$ & $\begin{array}{c}\text { Divide year, season or day into specific time blocks [7], } \\
\text { and specify tariffs according to different methods, such as } \\
\text { security-constrained unit commitment [16], Stochastic } \\
\text { Optimization Approach [17], [18], Monte Carlo simulation } \\
\text { [19], nonlinear economic modeling [20] and multi- } \\
\text { objective optimization [21]. }\end{array}$ \\
\hline Pritical Peak & $\begin{array}{c}\text { Less } \\
\text { predetermined } \\
\text { variant, between } \\
\text { TOU and RTP }\end{array}$ & $\begin{array}{c}\text { Focus on end users and analyze responses of different } \\
\text { kinds of customers during peak periods in order to make a } \\
\text { suitable schedule, by means of statistic methods [22], [23], } \\
\text { agent-based modeling [24], regression modeling [25] and } \\
\text { mathematical optimization [26] }\end{array}$ \\
\hline Pricing & $\begin{array}{c}\text { Market prices } \\
\text { timely act on } \\
\text { customers }\end{array}$ & $\begin{array}{c}\text { The most effective strategy, change customer's profiles of } \\
\text { consumption by real-time wholesale price. The methods } \\
\text { include reinforcement learning [3], agent-based modeling } \\
\text { [27], network analysis [28] and so on. }\end{array}$ \\
\hline
\end{tabular}


Considering the similarity of the natural gas supply systems and the electric power grids, there is a huge potential capacity to use these DSM methods to improve the reliability and efficiency of natural gas supply systems subject to consumption fluctuation. And more so, the recent significant progress on big data analytics and smart technology for application to natural gas supply systems can provide support to develop effective DSM strategies in natural gas supply systems [29], [30]. Unfortunately, the DSM strategies of smart electric power grids cannot be directly transferred to the natural gas supply systems, because [31], [32]: (1) natural gas is a compressible fluid, presenting transient behaviors in the pipeline transportation process; (2) because of the compressibility, natural gas can be stored in pipelines and create a "buffer area", which is quite different from power grids. Limited by these issues, the adjustments of system operation and resources allocation are often carried out based on off-line simulations, even though the current pipeline network systems already have good abilities to perform on-line monitoring and control [33]. Hence, besides the classical abilities of DSM, a feasible DSM strategy for natural gas supply systems also requires (1) accurate on-line prediction module of dynamic conditions of the complex gas pipeline networks; (2) evaluation of the short-term supply reliability with due consideration of the compressibility of the natural gas.

Considering the specific issues of natural gas supply systems, in this paper we develop a novel data-driven Real-Time Pricing method which can be used in the future smart gas supply systems to smooth customers' consumption fluctuation, improve supply reliability, increase the profits of the supply side and the satisfaction of the customers side. The developed DSM method mainly includes six parts: the hourly natural gas demand forecasting part, the system dynamic condition prediction part for complex gas pipeline networks, the supply reliability evaluation part, the customer behavior 
analysis part, the multi-objective optimization part and a final decision-making part. The main contributions of this work are summarized as follows:

(1) A novel systematic data-driven DSM method is developed for complex smart natural gas supply systems. Besides the classical properties of the DSM methods for electric power smart grids, the unique issues in natural gas supply systems, i.e., transient behaviors in the pipeline transportation process and line-pack storage, are considered in the development of DSM framework. As the best knowledge of the authors, this is the first time that the properties of natural gas pipeline network is considered in the research on DSM, which can provide new theoretical support for DSM in Integrated Energy Systems.

(2) A data-driven framework for natural gas supply system management is proposed. Advancing the classical management methods based on off-line simulation, the proposed framework overcomes the limitation of the strict requirements of accuracy of system description, boundary condition and initial condition, which enable the real-time management in complex natural gas pipeline networks, based on online data. This can provide interesting perspectives for the introduction of smart technologies into natural gas supply systems.

\section{Methodology}

\subsection{DSM framework for smart natural gas supply systems}

The general framework of DSM method can be sketched as in Fig. 1. Firstly, a prediction model, a recurrent neural network, $\mathrm{RNN}$ [34], in this paper, is used to forecast the demand of customers in future hours, based on the real-time data collected via smart metering at the demand side. Then, the forecast results are input to the customer response analysis model to predict the change of gas consumption in a specific time horizon, under a given price of gas. The results of the customer 

price.

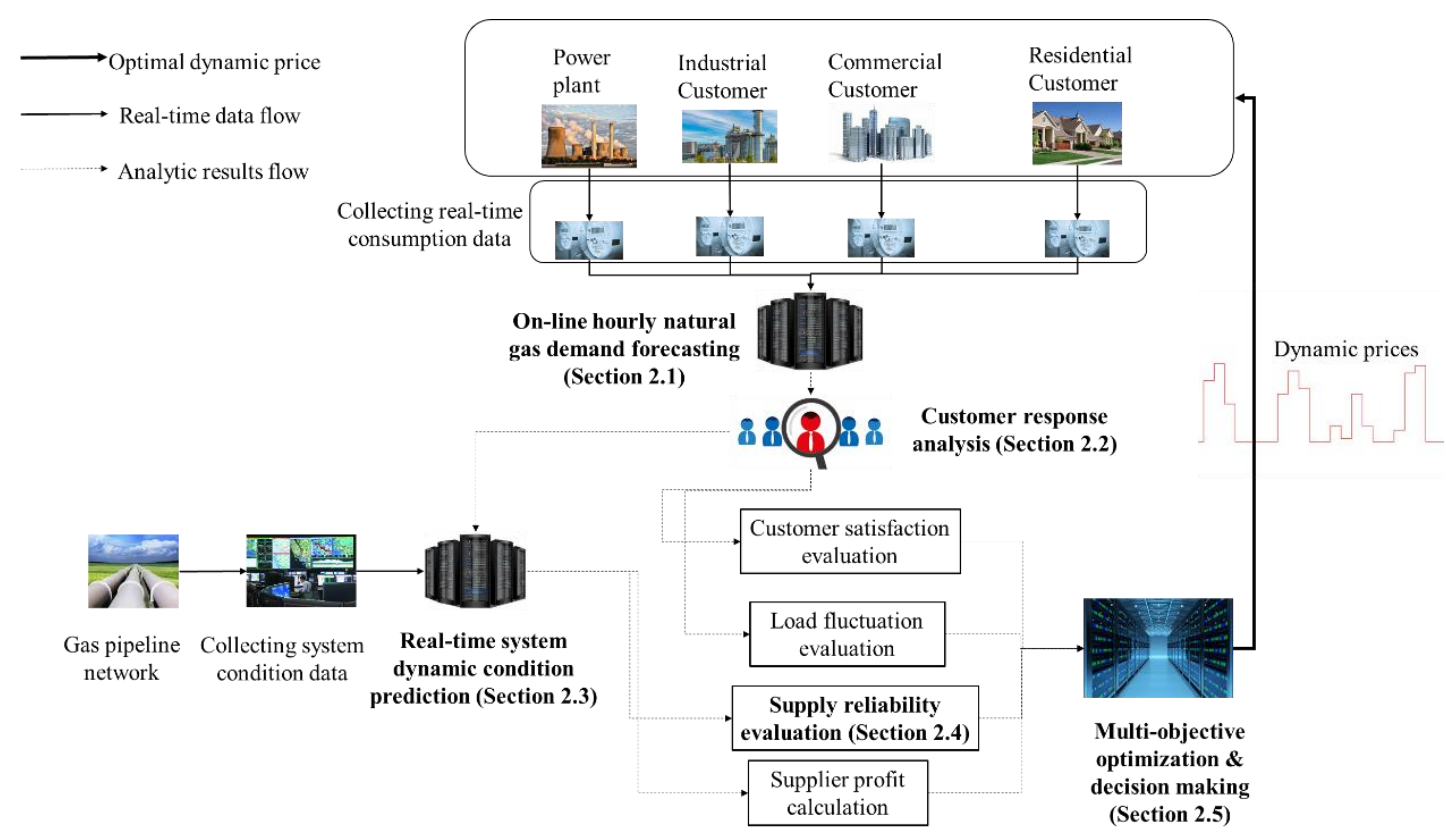

response prediction are combined with the real-time data of the pipeline network running conditions collected by the sensors, as input to the prediction model of the dynamic behavior of the complex pipeline network system. These corresponding prediction results, including delivery pressure at the demand sides and the outputs of the suppliers, are used to evaluate the supply reliability (based on the developed assessment method) and the profits of suppliers. Finally, the objectives of gas consumption fluctuation minimization, supplier profits maximization, supply reliability maximization and customer satisfaction maximization are integrated as a multi-objective optimization problem, here solved by the NSGA-II algorithm [35], [36]. Considering the complexity of the solution structure, decision-making rules are finally developed to find an optimal dynamic

Fig. 1 DSM framework for smart natural gas supply systems

\subsection{The natural gas demand forecasting model}

Accurate gas demand forecasting is essential to the effectiveness of the DSM strategy. The researches of energy demand forecasting have explored different kinds of methods, including time 
133 forecasting. Besides that, the complex relationships inside the demand data also add difficulty. Here,

series model [5], [37], regression model [38], [39] and artificial neural network [40]-[43]. Recently, some hybrid forecasting methods, integrating different models to overcome the relevant problems of the different, individual perspectives, have been proposed, giving better performance in terms of flexibility, computing efficiency, robustness, than the individual forecasting methods [30], [44], [45]. In this paper, we can firstly decompose the problem of natural gas demand forecasting into several sub-problems and, then, select proper methods to overcome them, respectively. Usually, natural gas demand data is complicated time series data, which significantly increase the difficulty of we develop a RNN-based hybrid forecasting method, as shown in Fig. 2: 


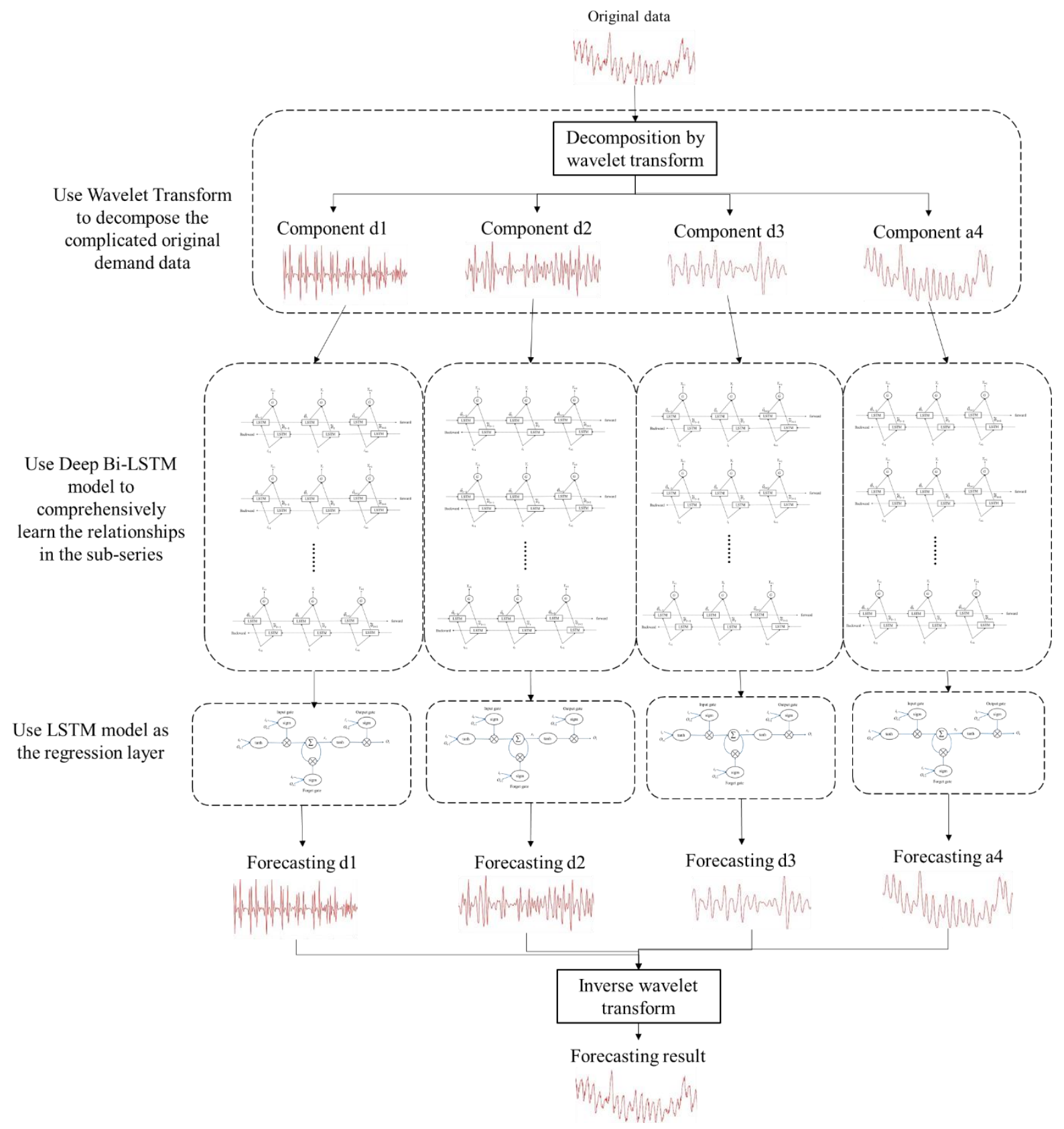

Fig. 2 Natural gas forecasting hybrid method

The Long Short Term Memory, Bi-LSTM and LSTM [46], models need to be pre-trained based

145 on historical consumption data and should, then, be updated periodically. Real-time natural gas

147 The wavelet transform [48], [49] used here can decompose the complicated original real-time gas

148 demand data into relatively simpler sub-series, which can effectively reduce the difficulty for the 
the patterns in the data because of the proved good performance of prediction on sequentialstructured data [50]. However, the traditional RNN models with shallow structure have presented limited capacity on large-scale, complicated data [51]. Hence, in this work, we use a deep Bi-LSTM model (shown in Fig. 3) to comprehensively learn the relationships among the gas consumption data from bi-directions, i.e., considering that the gas consumptions are influenced by both customers' habits and the regulation of the government. Then, a LSTM layer is put on the top of the deep BiLSTM model as a regression layer, to predict the future sub-series values based on the learned data features. Finally, the forecasted sub-series are integrated together by the method of reverse Wavelet Transform [30], to give the forecasting results of the natural gas consumption.

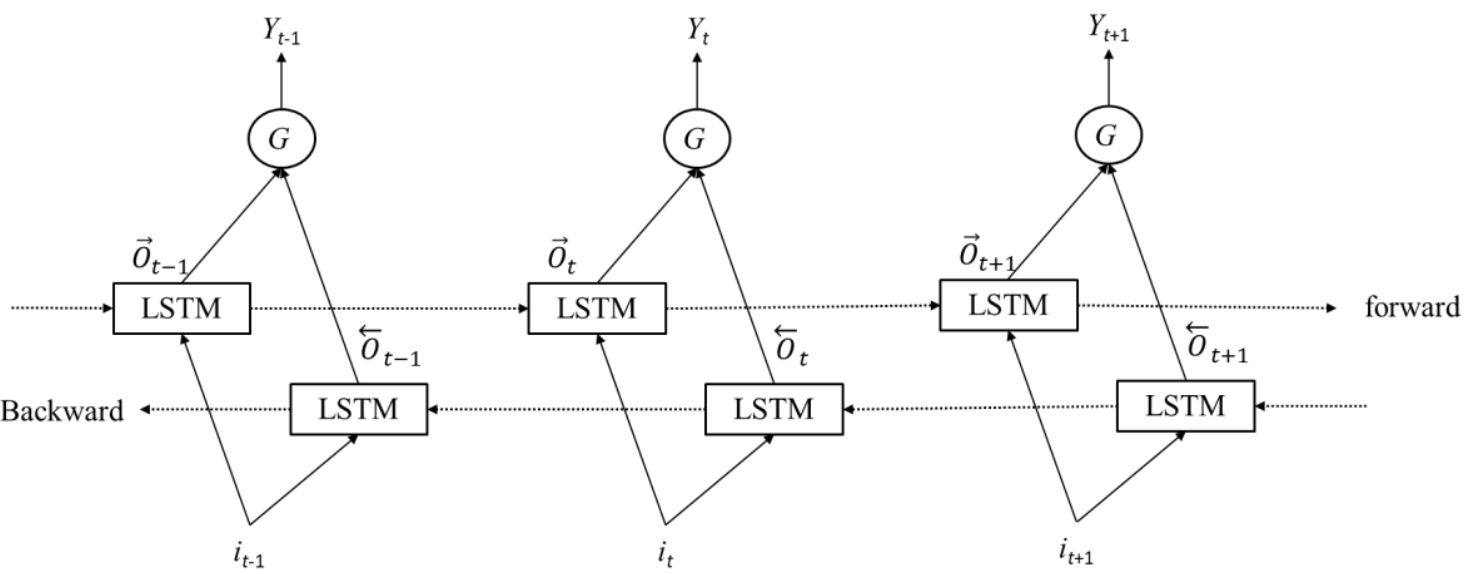

Fig. 3 Unfolded structure of a Bi-LSTM model

The developed forecasting model can capture and learn the complex relationships among the natural gas demand data, but the learned relationship sometimes are just noises. This problem of overfitting would be a serious problem, which can significant degenerate the forecasting accuracy. For this, we adopt the dropout technique to address the potential overfitting problem. The dropout technique is able to effectively prevent overfitting via randomly dropping units [52].

\subsection{The customer response model for consumption analysis}

One of the critical points in DSM is that the customers will act differently to different prices, 
which can be described by the price elasticity of demand in Microeconomics [53]. Based on that, the regulation center can use dynamic natural gas prices to stimulate the customers to change their patterns of consumption, to realize the target of gas load smoothing.

According to this concept, the basis of the dynamic price incentive on the customers is not only "consume more gas" or "save more money", but "get more feeling of happiness" by using natural gas. In economic theory, this kind of feeling is usually quantified by the value of Utility [54], [55]. In this work, we simplify the problem by assuming that happiness is generated by both using gas and saving money. Hence, the Utility function can be derived in the form of Equation 1, adopted from the reference [56].

$$
U c_{t+1}=-S_{t+1}-p r_{t+1} \cdot l_{t+1}
$$

in which $U c_{t+1}$ denotes the Utility of the customer at time $t+1$, which needs to be maximized; $s_{t+1}$ denotes the satisfaction of the customer by using natural gas; $p r_{t+1}$ denotes the price of natural gas at time $t+1 ; l_{t+1}$ represents the customer's consumption of natural gas at time $t+1$. The satisfaction $s_{t+1}$ can be calculated by Equation 2:

$$
s_{t+1}=d_{t+1} \beta_{t+1}\left[\left(\frac{l_{t+1}}{d_{t+1}}\right)^{\alpha_{t+1}}-1\right]
$$

in which $d_{t+1}$ denotes the forecasted gas demand (Section 2.2) of the customer in the specific future hours; $\alpha$ and $\beta$ are calculated by Equations 3-4:

$$
\text { factor }_{\alpha, t+1}=\frac{1}{E_{t+1}}+1\left(\mathrm{E}_{t+1}<0\right)
$$

$$
\beta_{t+1}=-\frac{n}{\alpha_{t+1}}
$$


187

where $n$ represents the fixed price of natural gas, which can also be understood as the price without dynamic pricing strategy. $E_{t+1}$ is the demand elasticity of price at time $t+1$, which distinguishes between different types of customers and changes along with time. According to the Equations, the customer has a worse feeling of happiness when $s>0(l<d)$ than the normal condition of $s=0$. Hence, in Eq. 1, the satisfaction is given a negative sign.

To find out the response of the customers under a given real-time price $p r$ and a potential demand of $d$ (forecasted value), we consider the first order derivative of $U c$ at time $t+1$ :

$$
\frac{\partial U c_{t+1}}{\partial l_{t+1}}=0
$$

which can be transformed as:

$$
-\alpha_{t+1} \beta_{t+1}\left(\frac{l_{t+1}}{d_{t+1}}\right)^{\alpha-1}-p r_{t+1}=0
$$

Then,

According to the second order derivative of Equation 7 and the ranges of $\alpha$ and $\beta$, the diagonal elements of the Hessian Matrix are all negative, and the values of the off-diagonal elements are all equal to zero. This means that $l_{t+1}^{*}$ is the optimal response of the customer with the given real-time price $p r_{t+1}$ and the forecasted demand $d_{t+1}$. According to the rationality hypothesis in Microeconomics [57], the $l_{t+1}^{*}$ is assumed to be the customer's consumption of natural gas, in the time horizon set.

\subsection{Prediction model for dynamic conditions in complex natural gas networks}




\section{based on Deep Learning}

The responses of the customers will cause dynamic changes in natural gas pipeline networks,

because of the transient behaviors. The unsteady changes in the pipeline network increase the conditions and very detailed system descriptions [58]-[60]. It is very difficult, even impossible, to use the off-line simulations to realize the real-time dynamic pricing, because of their strict requirements of boundary conditions and system description, and the noises in the data collected by

4:

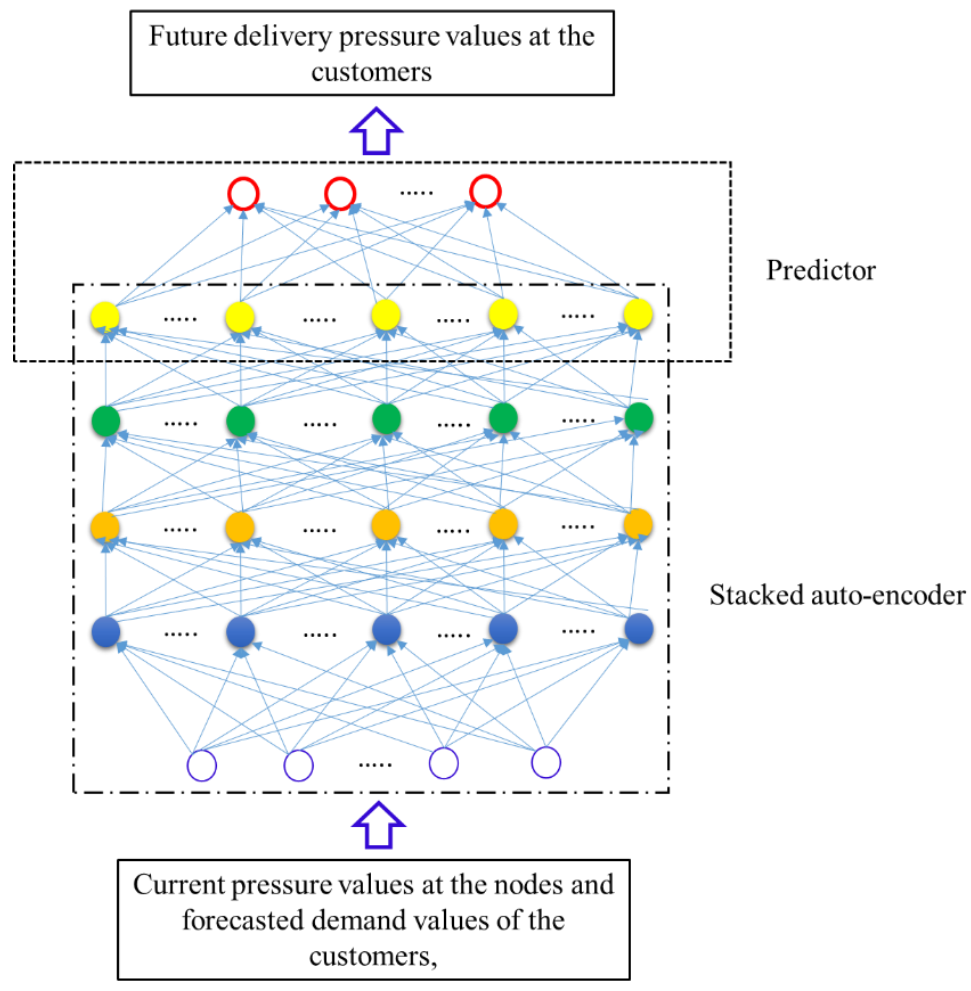

Fig. 4 Structure of the deep learning model for on-line condition prediction for natural gas pipeline 

demands at the customers, are preprocessed by data washing and normalization, as the input data of prediction. Then, these input data are fed into the deep neural network, which is constructed by stacking several auto-encoder models. According to the recent researches, the stacked auto-encoder model has a significant ability to capture dynamic properties in complex systems, such as power grids [63] and traffic systems [64]. Hence, here, we use the stacked auto-encoder model to learn the complicated patterns of the collected real-time data in the natural gas pipeline network, to improve the performance of the system condition prediction. The so-called auto-encoder model is a neural network that attempts to reconstruct the input signal at its output layer, after passing through hidden layers [65]. A sample auto-encoder model, with one input layer, one hidden layer and one output layer, is shown in Fig. 5. In the stacked auto-encoder model, the outputs from the lower hidden layer are taken as the inputs into the upper layer.

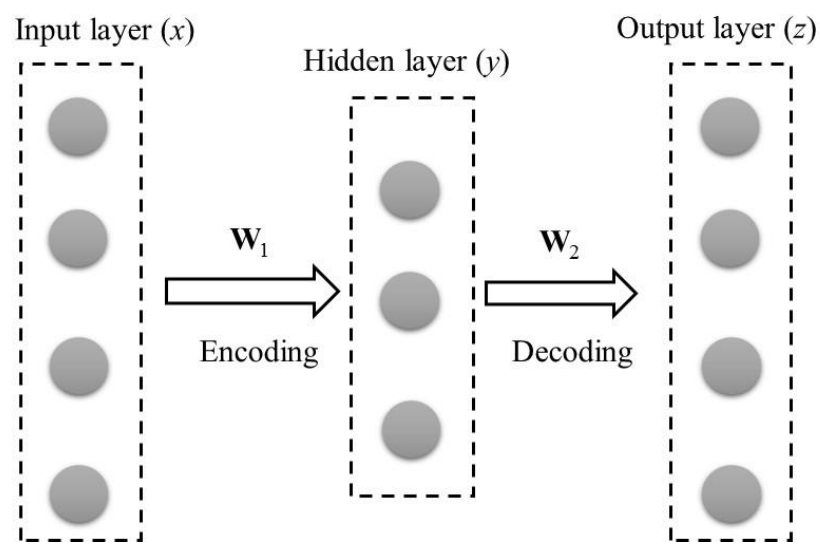

Fig. 5 A sample auto-encoder

To use the stacked auto-encoder model to perform the supervised prediction, a standard predictor is put on the top of the stacked auto-encoder model. In this work, a logic regression layer [66] is used as the predictor. In the supervised prediction, the delivery pressure in the next time step 
demand side which is closely related to the delivery pressure. In the next section, the predicted delivery pressure values are used as the critical parameters for the short-term supply reliability analysis.

\subsection{Supply reliability analysis method for natural gas pipeline systems}

The researches of DSM in electric power grids focus on the balance between supply and demand for analyzing the security of energy supply. However, the supply reliability in natural gas pipeline networks involves more than the balance: the compressibility of natural gas and the transient behaviors during transportation make the problem far more complex than balance. Therefore, in the DSM of natural gas supply systems, we need to consider such property of the gas pipeline systems in the compromise between reliability and efficiency for the DSM strategy.

In the short-term perspective, the supply reliability can be converted to a problem about maintaining a delivery pressure higher than the minimum contracted pressure of the customers. Hence, when determining dynamic prices, we need to consider to retain a reasonable pressure buffer under the influences of customers' responses, the dynamic properties of natural gas pipeline networks and their uncertainties.

In this section, a limit state function [67] is developed to evaluate the supply reliability of natural gas. The basic of this limit state function is the Mass Conservation Equation (Equation 8 below), because the hydraulic characteristic of natural gas pipeline systems is essential for this problem.

$$
\frac{\partial \rho_{\mathrm{n}}}{\partial t}+\frac{\partial\left(\rho_{\mathrm{n}} v\right)}{\partial x}=0
$$


demands and the connected delivery pressure. Considering this, Equation 8 is transformed into Equation 9 at the node of Demand $i$, connecting Pipeline $j$ :

$$
\frac{d p_{i}}{d t}=-\frac{\rho_{n} c^{2}}{\sum_{j=1}^{k} A_{i, j} \Delta x_{i, j}}\left(\sum_{j=1}^{k} Q_{i, j}-L_{i}\right)
$$

in Equation 9, $L_{i}$ represents the gas downloaded from $\left(L_{i}>0\right)$ or uploaded to $\left(L_{i}<0\right)$ the pipeline network. $Q_{i, j}$ represents the gas flow into delivery node $i$ from the connecting pipeline $j$. When gas flows from the pipeline $j$ to the delivery node $i, Q_{i, j}$ is negative; otherwise $Q_{i, j}$ is positive.

To clearly present the relationship between the delivery pressure and the demand, Equation 9 is transformed into Equation 10:

$$
p_{i, \text { potential }}=-\frac{\rho_{n} c^{2} \Delta t}{\sum_{j=1}^{k} A_{i, j} \Delta x_{i, j}}\left(\sum_{j=1}^{k} Q_{i, j}-L_{i}\right)+p_{i, \text { prediction }}
$$

in which $p_{i, \text { prediction }}$ represents the predicted delivery pressure in Section 2.4 and $p_{i, p o t e n t i a l}$ represents the delivery pressure after the time interval $\Delta t$, with the line-pack consumption rate of $\left(\sum_{j=1}^{k} Q_{i, j}-L_{i}\right)$

Generally, the variables in the pipelines should be calculated by the combination of the Mass Conservation Equation and the Momentum Conservation Equation, and the iteration process is very time-consuming. But according to the traditional concept of reliability [68], the supply reliability is closely related to uncertainties and it is unnecessary to solve the exact value of these variables in the analysis of reliability. For this reason, we hypothesize that the customer consumption $L$ and the flow rate $Q$ are stochastic variables and, then, develop a limit state function (Equation 10) to predict the potential risk of supply shortages. Here the uncertainties of the flow rates in the pipelines 
represent the uncertainties of the system supply capacity, due to the uncertain events in the pipeline systems, e.g., pipeline failures, maintenance behaviors or third-party damages.

$$
g_{i}\left(Q_{i j}, L_{i}, P_{i, t}, P_{\text {lim }, i}\right)=K m\left(\sum a_{i j} Q_{i j}-L_{i}\right) \Delta t+p_{i, \text { prediction }}-P_{\text {lim }, i}
$$

where $P_{\text {lim }, i}$ is the customer contracted minimum delivery pressure; $g_{i}<0$ represents the supply shortage at Customer $i$; the factor $K$ is calculated by Equation 12:

$$
K m=-\frac{\rho_{n} c^{2}}{\sum_{j=1}^{k} A_{i, j} \Delta x_{i, j}}
$$

where $\Delta x_{i, j}$ is the length of the part of Pipeline $j$ influenced by the gap between the delivered amount of gas and the demand of Customer $i ; \Delta t$ is the average duration of time that the consumption of the line-pack capacity may continue.

In many works, the limit state functions are solved by large repearted Monte-Carlo simulations, which is, to some extent, impossible for real-time DSM. Considering that, in this work, the limit state function is conveniently developed as a linear function of the stochastic variables, by which the probability of gas shortage can be directly calculated by Equations (13)-(14):

$$
\begin{gathered}
\operatorname{Pr}\left[g_{i}<0\right]=\Phi\left(-\beta_{i}\right) \\
\beta_{\text {demand }, i}=\frac{K\left(\sum a_{i j} \mu_{Q_{i j}}-\mu_{L_{i}}\right) \Delta t-\mu_{P_{\mathrm{lim}, i}}+p_{i, \text { prediction }}}{\sqrt{K^{2}\left(\sum a_{i j}{ }^{2} \sigma_{Q_{i j}}^{2}-\sigma_{L_{i}}^{2}\right) \Delta t-\sigma_{P_{\mathrm{lim}, i}}^{2}}}
\end{gathered}
$$

where $\mu$ and $\sigma$ denote the mean values and the variances of the stochastic variables.

In some conditions, probabilities of shortage are not sufficient to represent the target of reliability. Referring to the concept of risk, the consequences of shortages are also a critical element 
296

297

[69]. Generally, such consequences are quantified based on economic loss, which may be not suitable for the risk assessment of supply in large natural gas transmission networks. For example, we cannot compare the severity of natural gas shortages between an industrial province and a country's capital, only according to the amount of GDP. Considering that, we use the Utility concept [70] to quantify the loss of natural gas shortages for different customers, by introducing the quadratic utility function [71]:

where $v_{i, t}$ and $k_{i, t}$ are positive parameters which can be set, for example, by experts' opinion. $U_{i}\left(L_{i}\right)$ represents the utility of the Customer $i$ for the demand of $L_{i}$. The quadratic function is always used as utility function considering its non-decreasing property and the non-decreasing corresponding marginal benefits [70].

Hence, the risk of natural gas shortage $(S R)$ of Customer $i$, under the pressure $p_{i, p r e d i c t i o n}$, can be calculated by Equation 16 below:

$$
S R_{i}=U_{i} \Phi\left(-\beta_{\text {demand }, i}\right)
$$

The risk of natural gas shortage of the overall system, under the given dynamic price and the forecasted demands, is the summation of all customer risks:

$$
S R_{\text {system }}=\sum U_{i} \Phi\left(-\beta_{\text {demand }, i}\right)
$$

\subsection{Multi-objective dynamic pricing strategy}

In the dynamic pricing process, it is important to simultaneously deliberate multiple factors related to the interests of the different stockholders, including improving the profits of the suppliers, 
enhancing the supply reliability of the natural gas pipeline network, smoothing the fluctuation of gas consumption and increasing the happiness of the customers. Hence, the dynamic pricing problem is naturally converted to a multi-objective optimization problem, whose targets include:

(1) Maximization of the suppliers' profits:

$$
\max _{p r_{t+1}}\left\{U s_{t o t a l, t+1}\left(p r_{t+1}\right)\right\}=\max _{p r_{t+1}}\left\{L_{t o t a l, t+1} \cdot p r_{t+1}-L_{t o t a l, t+1} \cdot \cos t_{t+1}\right\}
$$

(2) Minimization of the risks of natural gas shortages:

$$
\min _{p r_{t+1}}\left\{S R\left(p r_{t+1}\right)\right\}=\min _{\text {price }_{t+1}}\left\{\sum U_{i} \Phi\left[-\beta_{\text {demand }, i}\left(p r_{t+1}\right)\right]\right\}
$$

(3) Minimization of the total fluctuation of natural gas consumption:

$$
\min _{p r_{t+1}}\left\{f_{t+1}\left(p r_{t+1}\right)\right\}=\min _{p r_{t+1}}\left\{\sum_{i=1}^{n}\left[l_{i, t+1}\left(p r_{t+1}\right)-d_{\text {iaverage }}\right]^{2}\right\}
$$

(3) Minimization of the total satisfaction of the customers:

$$
\max _{p r_{t+1}}\left\{s_{t+1}\left(p r_{t+1}\right)\right\}=\min _{p r_{t+1}}\left\{\sum_{i=1}^{n} d_{t+1} \beta_{t+1}\left[\left(\frac{l_{t+1}\left(p r_{t+1}\right)}{d_{t+1}}\right)^{\alpha_{t+1}}-1\right]\right\}
$$

Besides those target functions, there is a latent optimization target of maximization the happiness of the customers, which has already be considered in the model of customer response analysis in Section 2.3.

The multi-objective pricing strategy complies with the following constraints:

$$
p r_{\min } \leq p r_{t+1} \leq p r_{\max }
$$

$$
\text { cost } \leq p r_{t+1}
$$

$$
l_{i, \min } \leq l_{i, t+1}\left(p r_{t+1}\right) \leq l_{i, \max }
$$


335 where price $_{\min }$ and price $_{\max }$ define the acceptable range of the natural gas price; cost represents the pricing problem can be described as in Fig. 6 . 


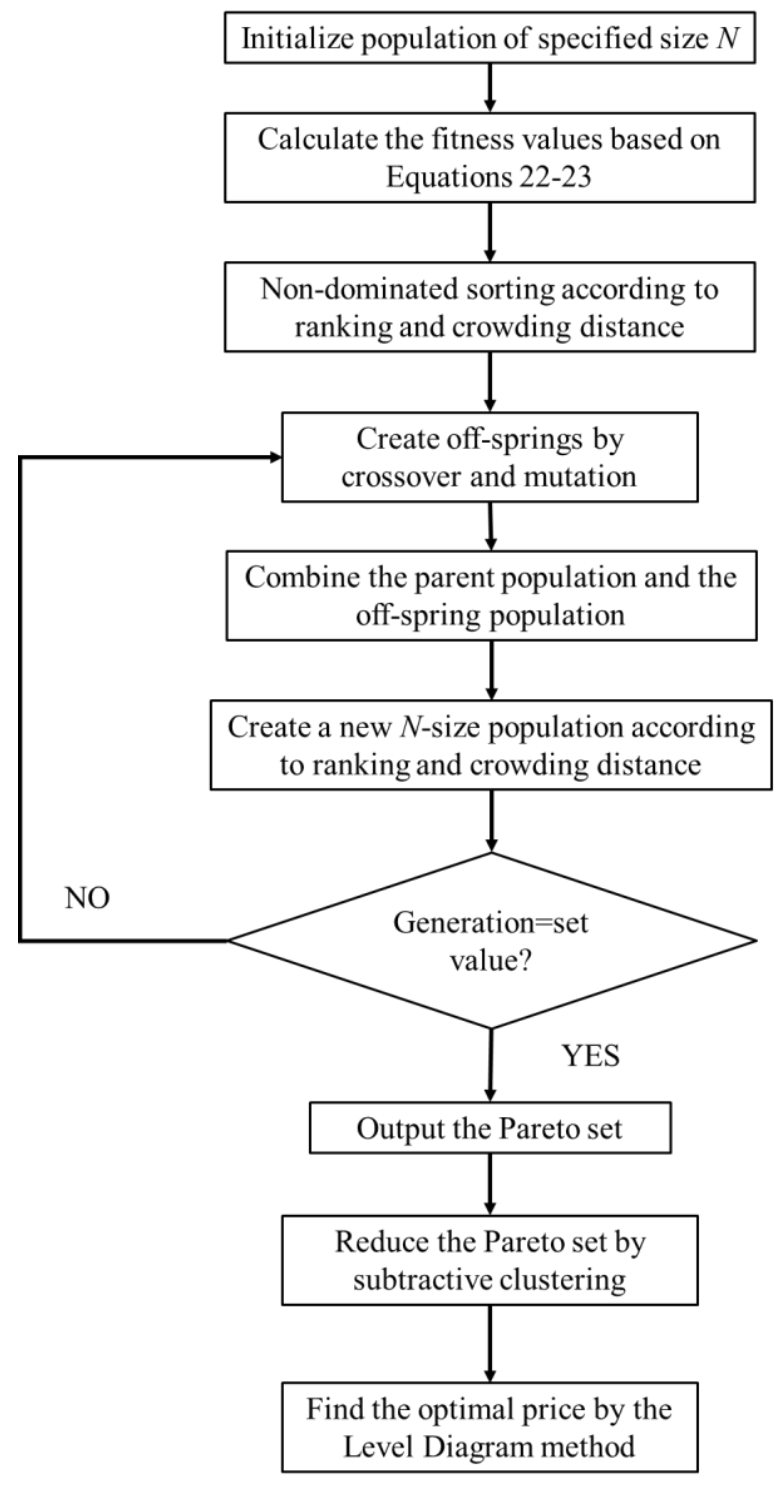

351 Fig. 6 The flowchart of the NSGA-II and the Level Diagram method to determine the optimal real-time price of natural gas

\subsection{Decision-making method for the optimal price}

Theoretically, all the solutions in the Pareto set can be used as a final decision for price making,

355 and the decision makers can select a preferred solution Here, we use a decision-making method combining the clustering method and the level diagram method to help selecting the optimal price from the Pareto set [74], [75]. 
them into a number of "families" (the number is $K$ ), according to their characteristics. In this work, the clustering method of subtractive clustering is applied to identify this kind of families [75], because of its advantages of independence on the choices of the initial cluster centers and the ability to directly represent the solutions on the Pareto set. Generally, clustering works is performed based on the distance between the elements which, in this work, is represented by the objective function values.

\subsubsection{Solutions reduction based on the subtractive clustering algorithm}

Assume a Pareto set $\Gamma$ including $n$ solutions, in which the $i$ th solution $\gamma^{j}$ represents a vector of objective values as follows:

$$
G\left(\gamma^{i}\right)=\left[G_{1}\left(\gamma^{i}\right), G_{2}\left(\gamma^{i}\right) \cdots G_{N U M o b}\left(\gamma^{i}\right)\right]
$$

in which NUMob denotes the number of objectives of the optimization problem. In this work,

NUMob is 4 . Considering that the four objectives are given in different scales and units, we need to firstly normalize them, e.g., to values between $[0,1]$.

$$
G_{j, \text { orm }}\left(\gamma^{i}\right)=\frac{G_{j}\left(\gamma^{i}\right)-G_{j, \min }}{G_{j, \max }-G_{j, \min }},
$$

Hence,

$$
G_{\text {norm }}\left(\gamma^{i}\right)=\left[G_{1, \text { orm }}\left(\gamma^{i}\right), G_{2, \text { norm }}\left(\gamma^{i}\right) \cdots G_{\text {NUMob,norm }}\left(\gamma^{i}\right)\right]
$$

Based on $G_{n o r m}$, we can calculate the potential $P\left(G_{n o r m}(\gamma)\right)$ for all the solutions in the set by:

$$
P\left(G_{\text {norm }}\left(\gamma^{i}\right)\right)=\sum_{l=1}^{n} e^{-\alpha \| G_{\text {norm }}\left(\gamma^{i}\right)-\left.G_{\text {norm }}\left(\gamma^{l}\right)\right|^{2}}
$$




$$
\xi=\frac{4}{r_{c}^{2}}
$$

379 where the cluster radius $r_{c}$ ranges from 0 to 1 and represents the range of influence of a cluster center

380 in every dimension. Hence, solutions outside $r_{c}$ should have negligible influences on the potential.

381 The value of the cluster radius will directly determine the number of clusters: a smaller value gives more but small clusters. According to the experience, the value of $r_{c}$ should be smaller than 0.5 , to maintain good clustering performances [75]. on this all the potential values of the other solutions are corrected as follows:

$$
P\left(G_{\text {norm }}\left(\gamma^{i}\right)\right)=P\left(G_{\text {norm }}\left(\gamma^{i}\right)\right)-P\left(G_{\text {norm }}^{1}\right) e^{-\beta\left\|G_{\text {norm }}\left(\gamma^{i}\right)-G_{\text {norm }}^{1}\right\|^{2}}
$$

in which,

$$
\zeta=\frac{4}{r_{c q}^{2}}, \quad r_{c q}=q \cdot r_{c}
$$
"neighborhood" solutions.

$$
P\left(G_{\text {norm }}^{j}\right) \geq \psi_{\text {accept }} P\left(G_{\text {norm }}^{1}\right)
$$




$$
P\left(G_{\text {norm }}^{j}\right) \leq \psi_{\text {reject }} P\left(G_{\text {norm }}^{1}\right)
$$

399

400

401

the cluster center is rejected and the process is over. $\psi_{\text {accept }}$ is the accept ratio and $\psi_{\text {reject }}$ is the reject ratio.

If Eq. 33 and Eq. 34 fail to find the cluster centers, one must introduce another acceptance criterion as follows:

$$
\frac{d_{\min }}{r_{c}}+\frac{P\left(G_{\text {norm }}^{j}\right)}{P\left(G_{\text {norm }}^{1}\right)} \geq 1
$$

in which

$$
d i s_{\min }=\min \left\|G_{n o r m}^{j}-G_{n o r m}^{m}\right\|, \quad m=1,2, \cdots j-1
$$

After all the cluster centers are found, the matrix $\tau$ of the membership function is generated via the standard Gaussian distribution:

$$
\tau_{j, i}=e^{-\xi\left\|G_{\text {norm }}\left(\gamma^{i}\right)-G_{\text {norm }}^{j}\right\|^{2}}
$$

Then all the solutions in the Pareto set can be assigned to the families, corresponding to the cluster centers, according to the membership function. The process of this subtractive algorithm shows no random initialization and the results are independent of the choices of the membership function and the initial cluster center. Besides, the obtained cluster centers are all corresponding to the Pareto solutions and can be directly picked as representative solutions of the reduced Pareto set [75].

\subsubsection{The decision-making method based on the Level Diagram}

After grouping the Pareto solutions into $K$ families, we still need to select the final optimal 
425 in which, $\bar{G}_{j}\left(\gamma^{i}\right)$, from 0 to 1 , can indicate the performance of decision $\gamma^{j}$ for the objective $j$.

solution for the decision. Level Diagram is an effective method to visualize the cluster representative solutions. It is based on the distance of the Pareto solutions to the ideal solution, considering the requirement of all the objectives of the problem. In a multi-objective optimization problem, with $m$ minimization objectives and $n$ maximization objectives, all the values of the objective functions can be normalized by:

$$
\bar{G}_{j}\left(\gamma^{i}\right)=\frac{G_{j}\left(\gamma^{i}\right)-G_{j, \text { min }}}{G_{j, \text { max }}-G_{j, \text { mix }}}, \quad j=1,2, \cdots, m
$$

$$
\text { or }
$$

$\bar{G}_{j}\left(\gamma^{i}\right)=0$ means the decision $\gamma^{j}$ is the ideal solution for the objective $j$; otherwise, it is the worst solution for the objective $j$.

$$
\bar{G}_{j}\left(\gamma^{i}\right)=\frac{G_{j, \text { mix }}-G_{j}\left(\gamma^{i}\right)}{G_{j, \max }-G_{j, \text { mix }}}, \quad j=1,2, \cdots, n
$$

To evaluate the deviation from the ideal solution, we need to choose a suitable norm, because different norms can give far different results from the same Pareto Front. In this paper, 1-norm (Eq. 40 ) is used considering its ability to simultaneously take into account every objective. Because in the DSM problem, the overall performance of a solution is critical for decision-making:

$$
\left\|G_{j}\left(\gamma^{i}\right)\right\|_{1-n o r m}=\sum_{j=1}^{N U M o b} \bar{G}_{j}\left(\gamma^{i}\right), \quad 0 \leq\left\|G_{j}\left(\gamma^{i}\right)\right\|_{1-n o r m} \leq j
$$

Then, the Level Diagram is drawn as follows: for each objective, the $X$ axis represents its physical value, while the $Y$ axis corresponds to the value of $\left\|G_{j}\left(\gamma^{i}\right)\right\|_{1-n o r m}$. The objectives are plotted separately. Hence, in the Level Diagram, the $Y$ axis is synchronized, and the $X$ axis presents 
the detailed information of the performances of the solutions on each objective.

In this work, we assume that the four objectives share the same importance of consideration of the decision makers. Solutions with lower norm values, which means they are closer to the ideal points of all the objectives, are preferred. Hence, the representative solutions in each clustered family are selected by:

$$
\left[F_{\text {optimal }}^{s}\right]=\min \left\|G_{j}\left(\gamma^{i}\right)_{f}\right\|_{1-n o r m}, \quad s=1, \cdots, K ; \quad f=1, \cdots, n_{s}
$$

where $K$ is the number of clustered families; $n_{s}$ denotes the number of solutions in the family $s$.

\section{Case study}

This section presents an application of the developed DSM framework to on a natural gas supply system. The system includes 36 pipelines (diameters ranging from $950 \mathrm{~mm}$ to $1014 \mathrm{~mm}$, total length of approx. $1100 \mathrm{~km}$ ), 23 customers, two compressor stations, five regulation stations, two pipeline importers, one LNG terminal and one underground gas storage (UGS). The UGS and the LNG terminal are set at flow rate-controlled modes, whereas the control modes of the two pipeline importers are pressure-controlled. The regulation stations are set as inactive modes. The pressures provided by the two compressor stations are maintained at the set points of $6.5 \mathrm{MPa}$ (Importer 2) and $7 \mathrm{MPa}$ (Importer 1), respectively. The gas pipeline network system is presented in

Fig. 7, where the customers are represented by the polygons. 


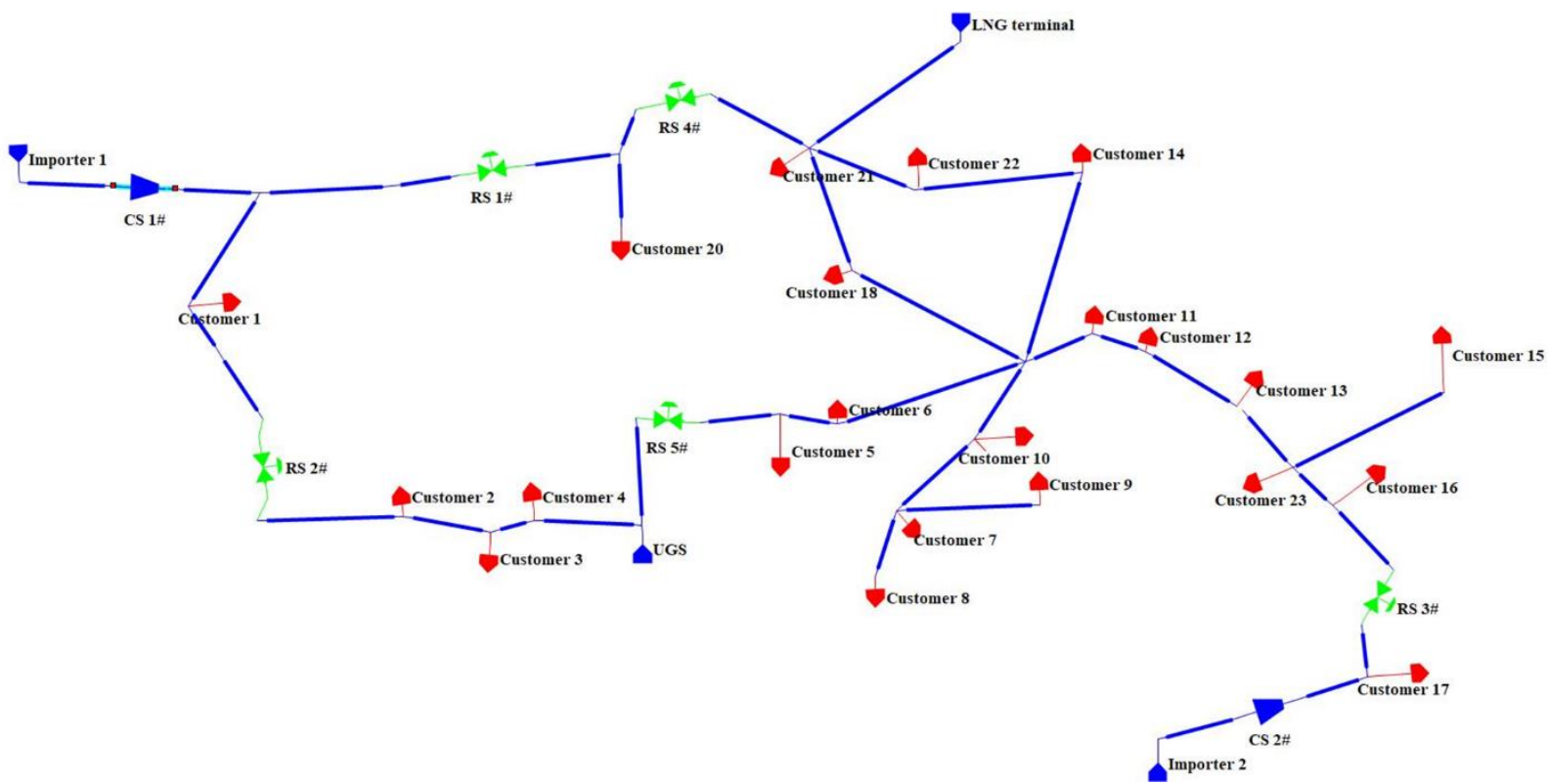

Fig. 7 Layout of the complex natural gas pipeline network system price of natural gas is set to 2.00 yuan $/ \mathrm{Nm}^{3}$, and the average cost of production and transportation is assumed to 1.60 yuan $/ \mathrm{Nm}^{3}$. But, we should notice that these parameters should be strictly determined by the collected data in the engineering applications, to obtain credible results. used to generate the natural gas consumption data. The Mackey-Glass model (Equation 26) is a periodic and chaotic time-series model, which is often used to verify the performance of predictive models [48]. Its chaotic behaviors are similar to the properties of fluctuations of natural gas consumptions, because the current data values are dependent on those of the past:

$$
\frac{d x(t)}{d t}=\frac{a x\left(t-\tau_{M}\right)}{1+x^{c}\left(t-\tau_{M}\right)}-b x(t)
$$


(>16.8), which determines the chaotic property of the generated time series. In this case, the value of $\tau_{M}$ has been set to be 20 . The $4^{\text {th }}$ Runge-Kutta method is used to generate the time series data and, then, the data is sampled at a given interval in our case equal to I hour. Besides, to make the application more realistic, a random term (of $1 \%$ of the nominal value of the generated gas demands) is introduced to the generated series data.

Then, we need a "real" environment to generate operational data to test the performance of the DSM method and generate the data to train the deep learning model in Section 2.4. Considering that, a commercial software TGNET, which is professional in steady state and transient thermal-hydraulic simulation of gas pipeline networks, is used here to simulate the system working conditions according to the following assumptions and principles:

(1) The active components are set at specific control modes, with desired set values;

(2) The system condition changes along with the fluctuations in demands. There are two types of boundary conditions, i.e., consumptions with DSM and consumptions without DSM. Therefore, system conditions based on the former are used as the verification and those based on the latter are the benchmark.

\subsection{Results and analysis}

Accurate demand forecasting is the fundamental part of this DSM framework, which can directly impact the effectiveness and the usability. Hence, firstly, the accuracy of the proposed forecasting model (in Section 2.2) is tested, based on the series data generated by the Mackey-Glass model. The number of layers of the Bi-LSTM model is set to 2, by trial and error considering both the forecasting accuracy and the time consumption of training. Besides the number of layers, the learning rate during the training process can also affect efficiency and performance. In this work, 
the adaptive moment estimation (Adam) optimizer is adopted to train the deep-RNN forecasting mode. The initial learning rate is set to 0.005 and a maximum number of epochs for training is 300 . In real-world application, a pre-processing step, e.g., data washing, should be performed prior to entering the data into the forecasting model. The whole data set is divided into $70 \%$ and $30 \%$, used to train and test the forecasting model, respectively. After the natural gas demand data is decomposed by the wavelet transformation, the values of the autocorrelation functions of every wavelet component, which are used to identify the series data periodicity, are calculated. Based on these values, the input sizes of the components are determined, as the lengths of their first periods which are the most relevant data for the forecasting. By this method, the training set is accordingly grouped into a number of sub-sets for the training process.

\section{The forecasting time interval of the presented applications is chosen as 10 hours, and the} forecasting results are presented in Fig. 8 for both the original data and the components:

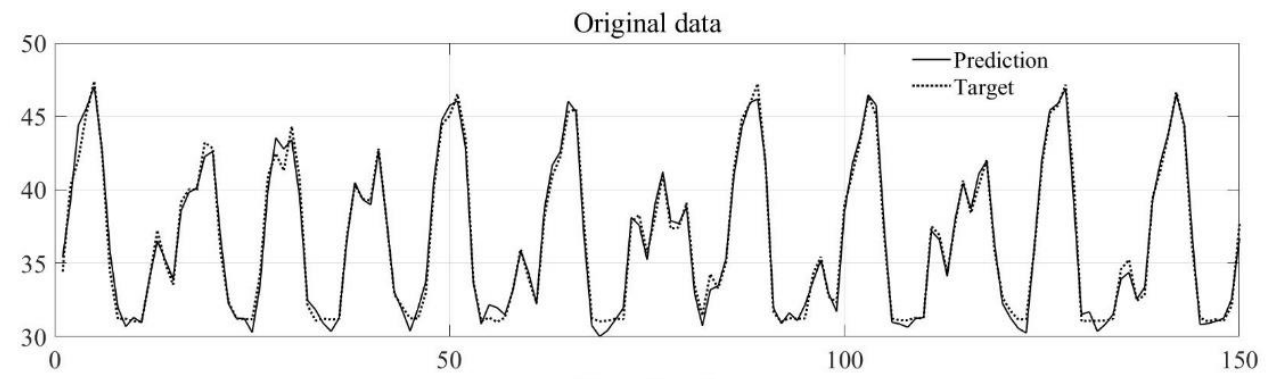

Time (hour)

Component $\mathrm{d} 1$

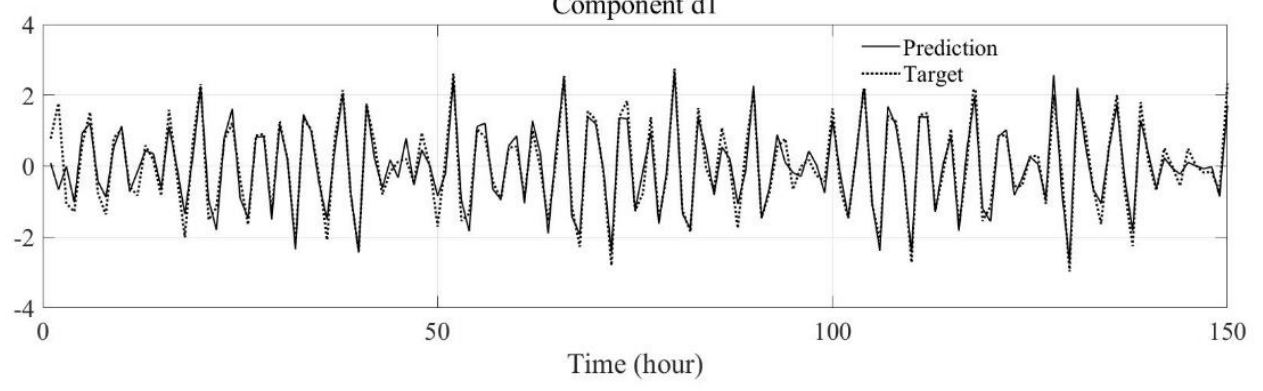



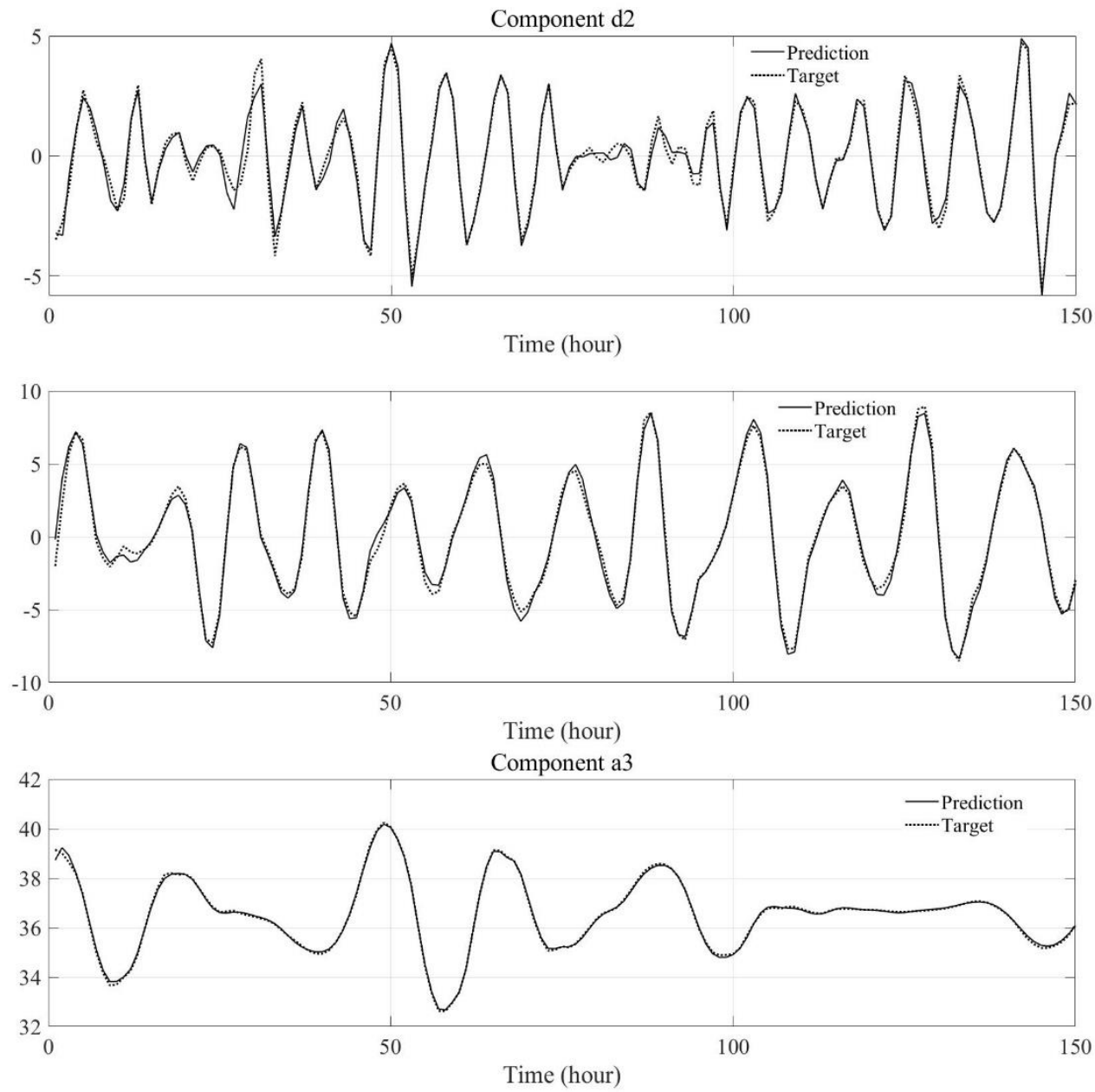

Fig. 8 Forecasting results on the Mackey Glass generated data

The forecasting performance is also quantified in terms of Mean Absolute Error (MAE), Mean

Relative Error (MRE) and Root Mean Squared Error (RMSE):

$$
\begin{aligned}
& M A E=\frac{1}{N} \sum_{i=1}^{N}\left|F_{i}-T_{i}\right| \\
& M R E=\frac{1}{N} \sum_{i=1}^{N}\left|\frac{F_{i}-T_{i}}{T_{i}}\right|
\end{aligned}
$$

$$
R M S E=\frac{1}{N} \sqrt{\sum_{i=1}^{N}\left(F_{i}-T_{i}\right)^{2}}
$$

where $F_{i}$ denotes the forecasting result and $T_{i}$ denotes the target value.

Table 2 Prediction performances on the Mackey Glass series for different forecasting horizons

\begin{tabular}{c|c|ccc}
\hline Data basis & Task & MAE & MRE & RMSE \\
\hline The Mackey & 1 hour forecasting & 0.0596 & 0.0017 & 0.1182 \\
\hline
\end{tabular}




\begin{tabular}{l|c|ccc}
\hline \multirow{2}{*}{ Glass series } & 5 hours forecasting & 0.1960 & 0.0054 & 0.5537 \\
\cline { 2 - 5 } & 10 hours forecasting & 0.5724 & 0.0157 & 1.0719 \\
\hline
\end{tabular}

Fig. 8 and Table 2 indicate a good performance of the developed model for forecasting natural gas demand. The performances in Table 2 show that the MRE, which can be used to represent the errors of the forecasting, remains around 0.0157 even though the prediction horizon is set to 10 hours ahead. Time complexity and space complexity are also important indices to evaluate the model. The time complexity of the proposed forecasting model is determined by the time complexity of each layer. The deep-RNN model is constructed by stacking two Bi-LSTM layers and one LSTM layer, with the time complexity of $\mathrm{o}(2 W)$ and $\mathrm{o}(W)$, which can be calculated follows:

$$
W=4 I_{\text {num }} H_{\text {nит }}+4 H_{\text {num }}^{2}+3 H_{\text {nит }}+H_{\text {num }} K_{\text {num }}
$$

in which $I_{\text {num }}$ denotes the number of inputs, $H_{\text {num }}$ denotes the number of hidden cells, $K_{\text {num }}$ denotes the number of outputs.

The space complexity $(S C)$ determines the memory storage needed by the algorithm, which, in LSTM and Bi-LSTM layers, can be represented by the number of their parameters:

$$
S C=4\left(I_{\text {nит }} H_{\text {nит }}+H_{\text {num }}^{2}+H_{\text {num }}\right)
$$

Comparing with the previous forecasting models based on the shallow neural networks, their time complexities are close. The space complexity of the proposed algorithm is, however, higher than the previous ones, which means more memory storage is needed. From another perspective, the larger number of parameters is, of course, also the reason of enabling the developed model to better fit the high nonlinearity of natural gas demand data.

In the DSM method, the responses of the natural gas pipeline network to the customers' behaviors, because of its dynamic property, can significantly influence the supply reliability and the 

gas pipeline network is also very important for making successful DSM strategies. The ability of the developed deep learning model has been verified in our previous work [61], for the prediction of dynamic changes in the gas pipeline network. the Pareto Front, which is made of 188 solutions.

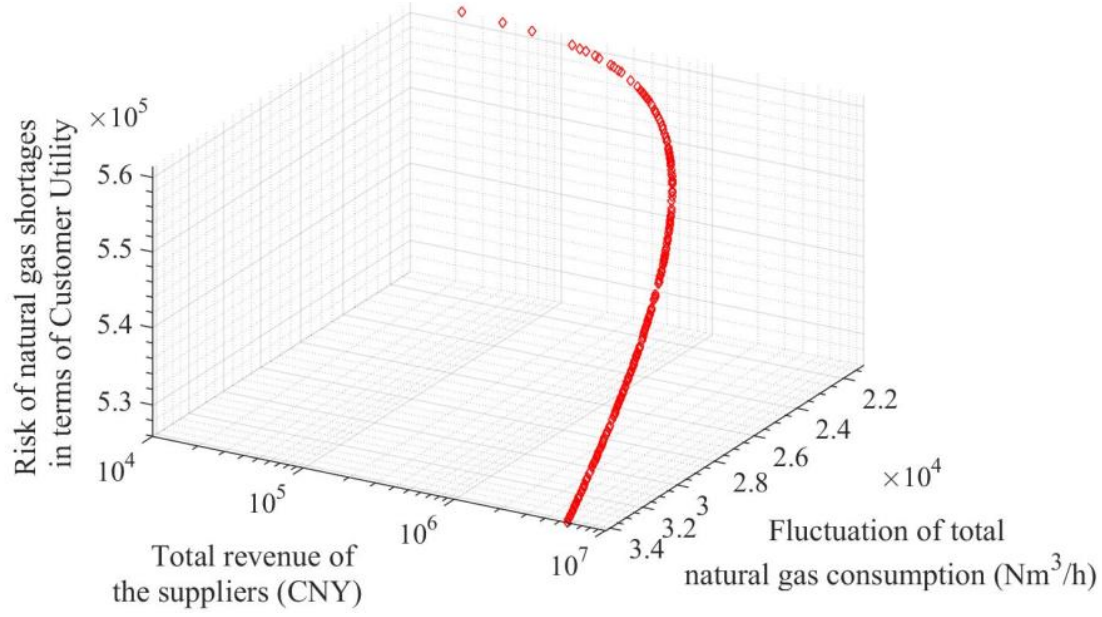

Fig. 9 Pareto Front of the multi-objective DSM problem

The Pareto solutions are obtained through the NSGA-II algorithm and all the solutions represent the compromises between different, sometimes, conflicting objectives. To analyze the relationships between the four objectives and illustrate the power of the decision-making method, the Level Diagram presentation is plotted in Fig. 10 before performing the subtractive clustering. 


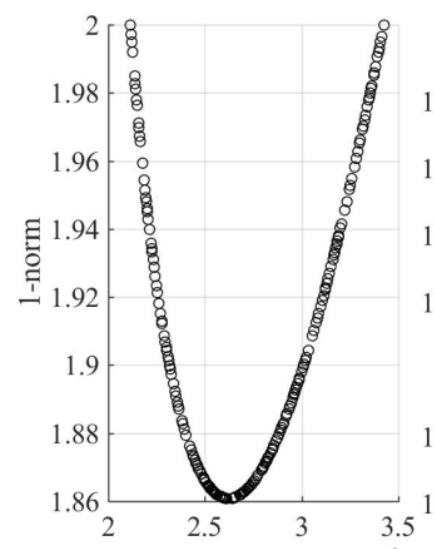

Fluctuation of total $\times 10^{4}$ natural gas consumption $\left(\mathrm{Nm}^{3} / \mathrm{h}\right)$

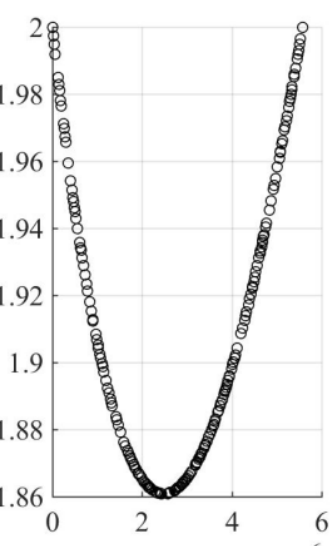

Total revenue of $\times 10^{6}$ the suppliers (CNY)

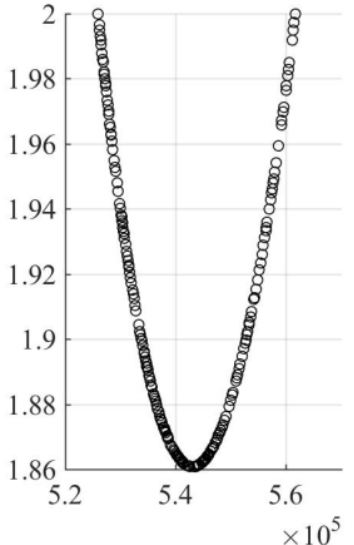

Risk of natural gas shortages in terms of Customer Utility

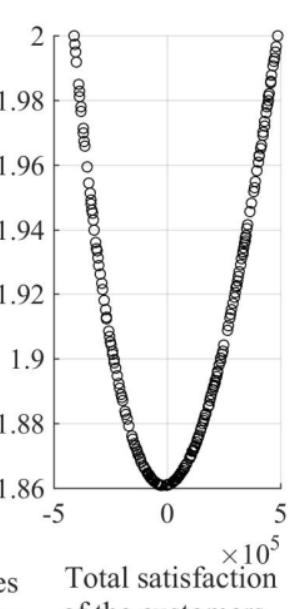

of the customers
551

552

553

554

555

556

557

558

559

560

561

562

563

Fig. 10 Level Diagram representation of the original Pareto Front of the multi-objective DSM problem

However, it is difficult to take a final decision and choose one preferred solution from the dense

Pareto Front and corresponding Level Diagram of Figs. 9-10. To this aim, the decision-making method introduced in Section 2.7 is used to reduce the Pareto solutions and select the optimal price of gas.

The value of the cluster radius can significantly influence the performance of the clustering. In this paper, the radius is determined by trial and error with referred to the global silhouette criterion [75], which is computed as follows:

$$
G S=\frac{1}{K} \sum_{i=1}^{K} C S_{i}
$$

where $C S_{i}$ represents the cluster silhouette of the cluster family $i$, which can be calculated by the average value of the silhouette width $\operatorname{cs}(n)$ in the $i$ th cluster. The $c s(n)$ can be obtained by:

$$
c s(n)=\frac{b(n)-a(n)}{\max \{a(n), b(n)\}}
$$

where $a(n)$ represents the average distance from the solution $n$ to the other solutions in the cluster; $b(n)$ is the average distance from the solution $n$ to the solutions in the nearest cluster. The trial and error attempts are performed in the range of $[0.1,0.4]$, by steps of 0.01 . The cluster radius is finally 
determined to be 0.19 .

Fig. 11 presents the Pareto Front after clustering and Fig. 12 gives the Level Diagram representation of the reduced Pareto set. From the Figures, we can observe that the Pareto Front maintains the original shape but with significant reduction of the solutions, which are more intelligible and easier to handle for selecting the optimal prices in the DSM.

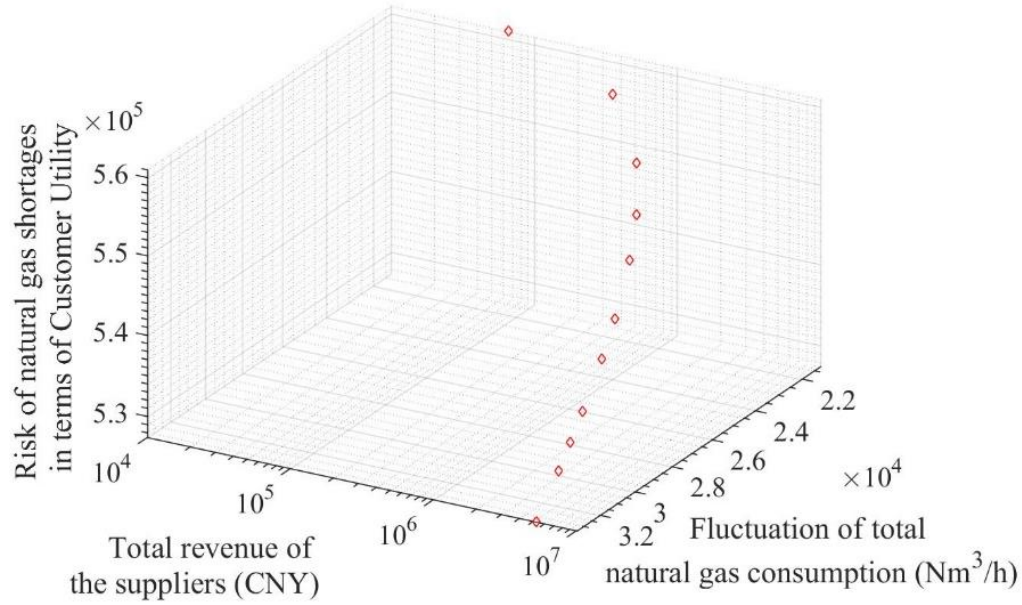

Fig. 11 Pareto Front of the reduced set of solutions of the multi-objective DSM problem

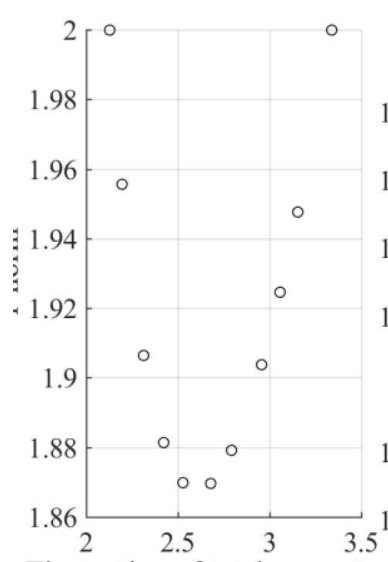

Fluctuation of total $\times 10^{4}$ tural gas consumption $\left(\mathrm{Nm}^{3} / \mathrm{s}\right.$

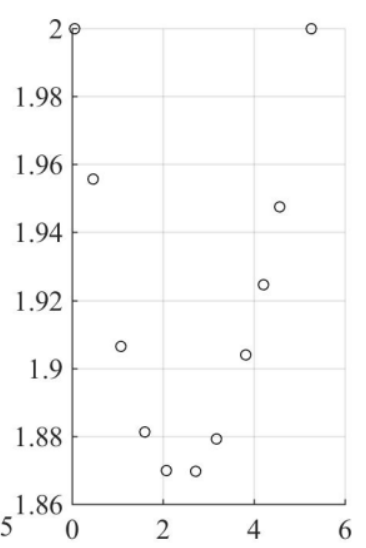

Total revenue of $\times 10^{6}$ the suppliers (CNY)
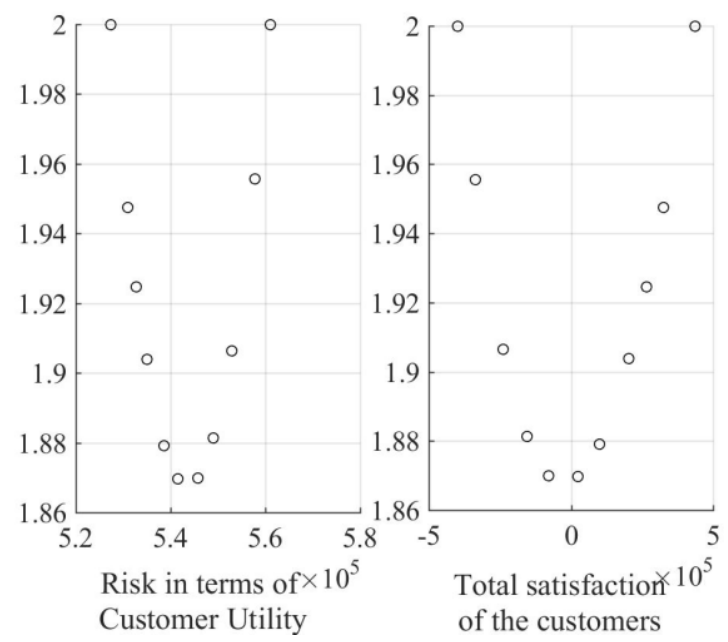

Fig. 12 Level Diagram representation of the family representative solutions, for the multiobjective DSM problem

Based on the simplified Level Diagram, the optimal solution can be easily found, which is corresponding to the final decision of the natural gas price. To verify the effectiveness of the multiobjective dynamic pricing strategy, this is performed based on different time horizons of pricing, 


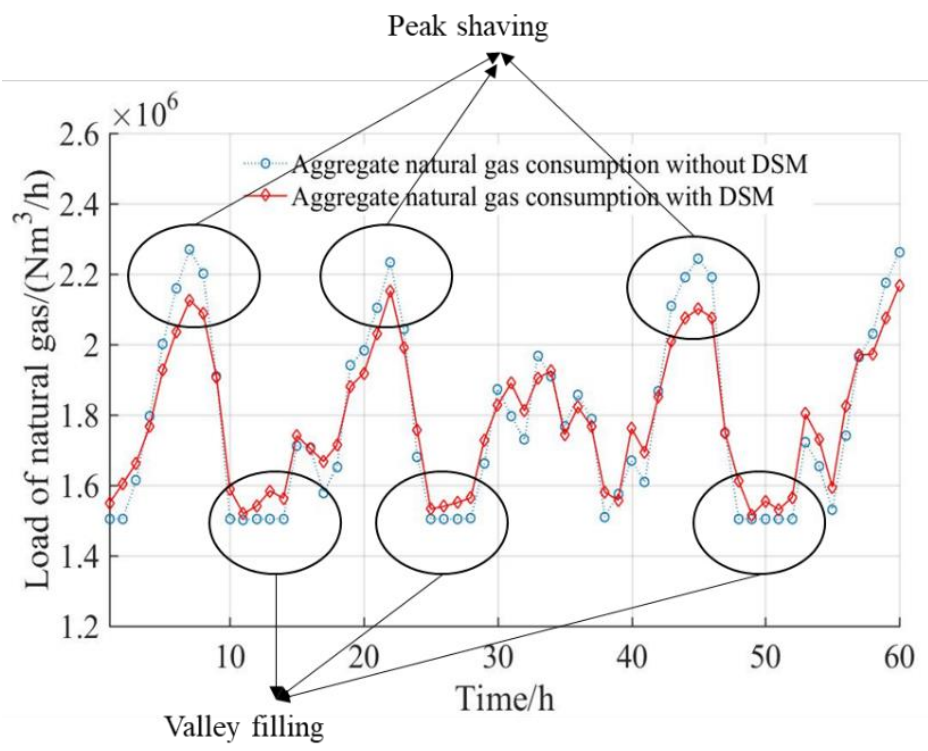

Fig. 13 Comparison of the aggregate natural gas consumptions, before and after performing the

DSM strategy (for 1 hour pricing period)

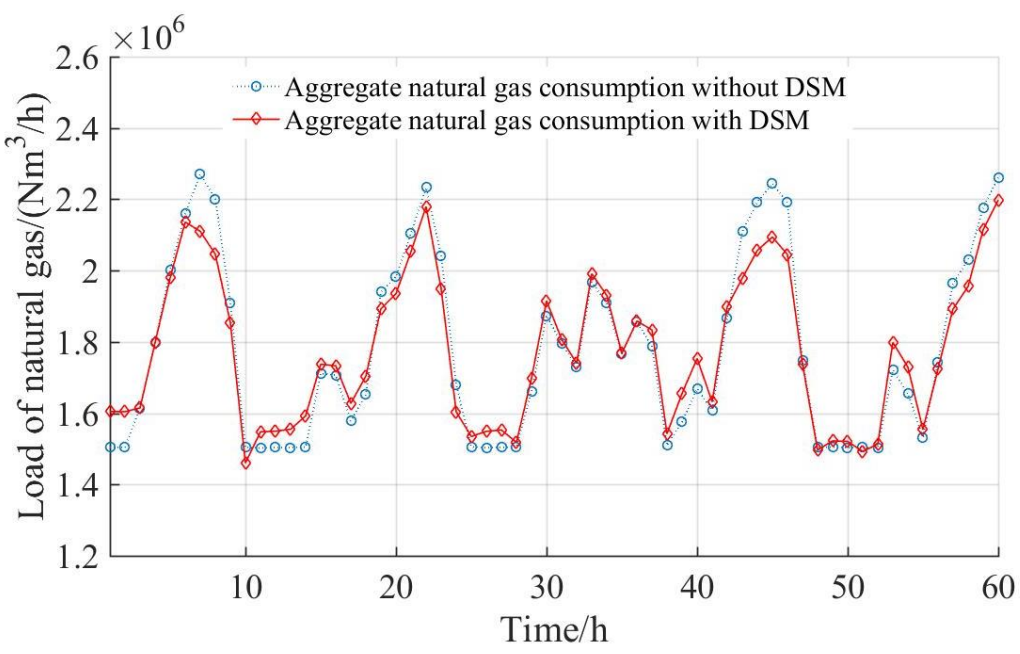

Fig. 14 Comparison of the aggregate natural gas consumptions, before and after performing the DSM strategy (for 2 hours pricing period) 


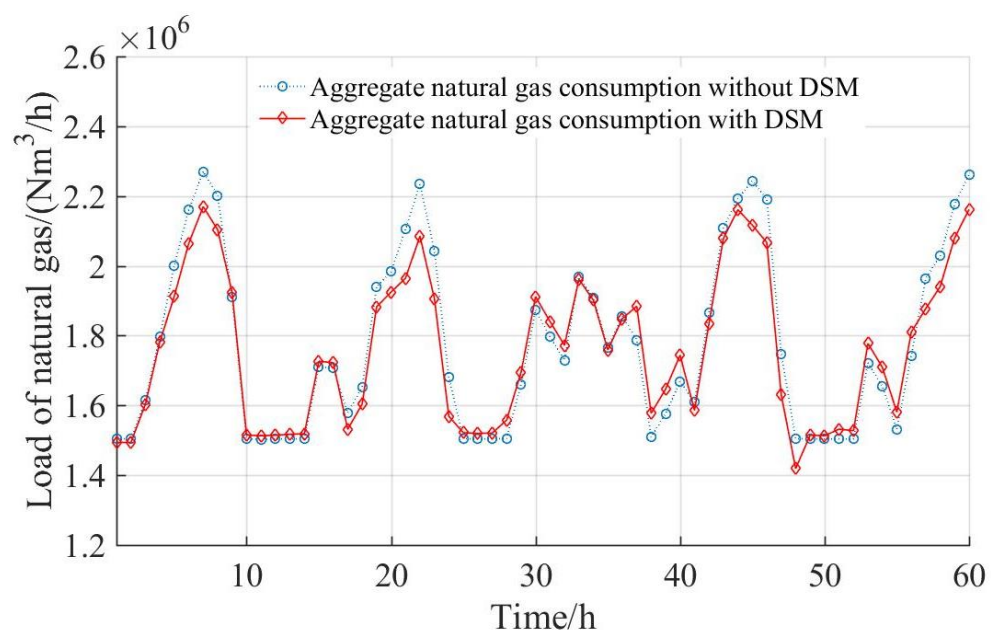

Fig. 15 Comparison of the aggregate natural gas consumptions, before and after performing the DSM strategy (for 4 hours pricing period)

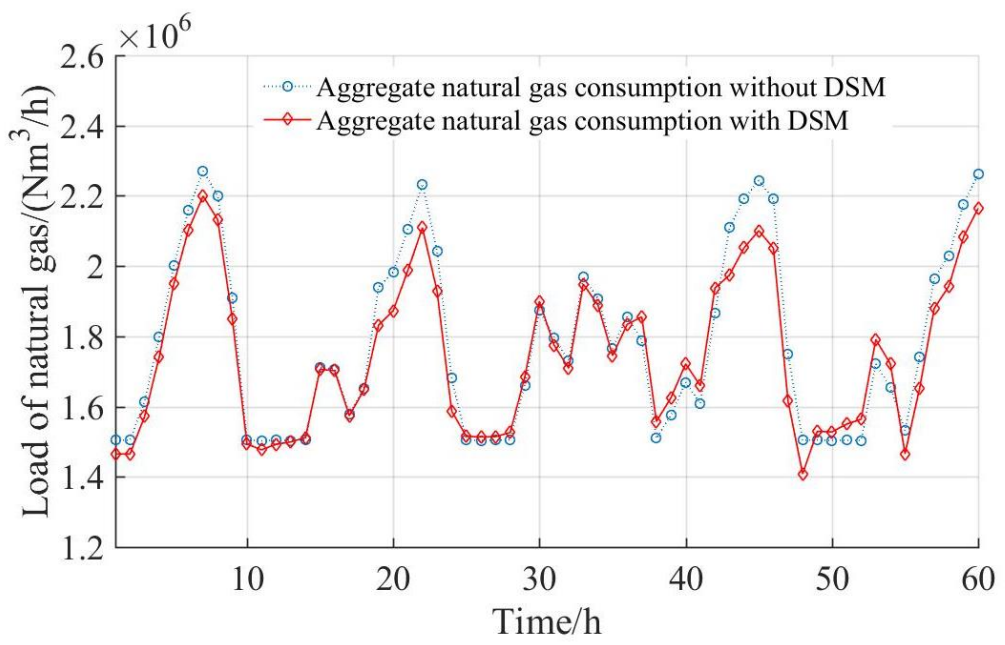

Fig. 16 Comparison of the aggregate natural gas consumptions, before and after performing the DSM strategy (for 6 hours pricing period)

Figs. 13-16 show that the developed DSM strategy has a relatively good performance of peak shaving and valley filling, for all four selected time periods of pricing. The results also indicate different performances on different pricing periods: the performances of the pricing periods of 1 hour and 2 hours are slightly better than those of the pricing periods of 4 hours and 6 hours. This is because of the higher flexibilities of the strategies with shorter pricing periods. From the overall perspective, the DSM shows a good stability on different pricing periods.

The following indices are calculated based on the results of the application of DSM, with respect to the other aspects of suppliers' profit, supply reliability and customers' satisfaction. The 
604

605

profit improvement $(P I)$ is calculated by Eq. 48, which represents the change in suppliers' profits after DSM with respect to a strategy of fixed price of the natural gas. The aggregate consumption fluctuation reduction $(A C F R)$ is introduced to quantify the effect of the dynamic pricing strategy on reducing the load fluctuation $(L F)$ of natural gas, which is calculated based on Eqs. 51-52. The peak reduction $(P R)$ represents the ability of the DSM method to shave the peak of load, which is calculated by Eq. 53. The performance of improving the supply reliability is quantified by Eq. 54, which represents the risk reduction $(R R)$ after DSM. Satisfaction is measured by aggregating all customers' satisfactions calculated in Eq. 2, which is named aggregate customers' satisfaction $(A C S)$ :

$$
P I=\frac{\text { profit }_{D S M}-\text { profit }_{\text {fixed_price }}}{\text { profit }_{\text {fixed_price }}}
$$

in which profit ${ }_{D S M}$ and profit $t_{\text {fixed price }}$ denote the total profits of the suppliers with and without DSM, respectively;

$$
\begin{aligned}
& A C F R=\frac{A C F_{D S M}-A C F_{\text {fixed_price }_{\text {_p }}}}{A C F_{\text {fixed_price }_{\text {_ }}}} \\
& A C F=\frac{1}{N} \sum_{t=1}^{N} \mid \operatorname{load}_{i}-\text { load }_{\text {average }} \mid
\end{aligned}
$$

in which $A C F_{D S M}$ and $A C F_{\text {fixed_price }}$ represent the aggregate consumption fluctuations with and without DSM, respectively. load $_{i}$ is the natural gas consumption at time step $i$ and load average $_{\text {is }}$ the average natural gas consumption;

$$
P R=\frac{1}{N P} \sum_{i=1}^{N P} \frac{\text { peak }_{D S M, i}-\text { peak }_{\text {fixed_price }, i}}{\text { peak }_{\text {fixed_price }, i_{i}}}
$$

in which peak $k_{D S M, i}$ and peak $k_{\text {fixed_price, } i}$ denote the $i$ th peak of loads with and without DSM, respectively. $N P$ is the number of peaks;

$$
R R=\frac{S R_{\text {fixed_price }}-S R_{D S M}}{S R_{\text {fixed_price }}}
$$


624 the DSM decreases.

in which $S R_{D S M, i}$ and $S R_{\text {fixed price, } i}$ are estimated by the method proposed in Section 2.5, and are the risk of shortage with and without the DSM, respectively.

The values are presented in the following Table 3.

Table 3 Performances of the developed DSM method

\begin{tabular}{cccccc}
\hline $\begin{array}{c}\text { Pricing } \\
\text { period }\end{array}$ & $P I(\%)$ & $P R(\%)$ & $A F C R(\%)$ & $R R(\%)$ & $A C S\left(\times 10^{6}\right)$ \\
(hour) & & & & & \\
\hline 1 & 0.67 & 6.38 & 21.43 & 40.10 & -1.05 \\
2 & 1.58 & 7.06 & 20.16 & 26.22 & -0.28 \\
4 & 6.81 & 6.72 & 15.13 & 28.15 & 1.77 \\
6 & 10.56 & 7.61 & 12.10 & 32.34 & 4.33 \\
\hline
\end{tabular}

The results in Table 3 show the good performances of the developed DSM framework for the different pricing periods considered. The DSM method is able to effectively reduce the peaks of load, around $6 \%-7 \%$ in all pricing periods, and significantly improve the short-term supply reliability of pipelines. According to the values of the index of $A F C R$, we can conclude that the approach is capable of smoothing the fluctuation of natural gas consumption, which can improve the efficiency of resources usage and system operation. In the case study, the horizons of 1 hour and 2 hours can be identified as the best choices of the pricing period for the dynamic pricing DSM. As to the pricing period of 4 hours and 6 hours, although they show satisfactory results for most objectives, however, the results of $A C S$ indicate that the customers may suffer (a positive $A C S$ value means a loss of satisfaction). This is because as the pricing period becomes longer, the flexibility of 


\section{Conclusions and future works}

In this paper, a systematic data-driven Demand Side Management framework is developed for future smart natural gas supply systems, with the targets of load profile smoothing, supply reliability enhancement, company profit improvement and customers' satisfaction. The framework can help the natural gas supply system improving the efficiency, reliability and flexibility. The DSM framework integrates five main parts, including demand forecasting, customer response analysis, real-time system dynamic conditions prediction, supply reliability evaluation, multi-objective optimization and decision-making.

The gas demand forecasting model is built up by combining wavelet transform and Deep-RNN model, for learning the complex patterns of gas consumption data and make accurate hourly forecasting of natural gas demand. The customer response analysis is performed based on Microeconomics theory, to analyze the behaviors of the customers according to their demand elasticity of price and the forecasted demand. Then, a deep-learning-based prediction model is used for the dynamic condition of natural gas pipeline networks, considering the changes of the system due to the customers' demand changes and the complex physical process of gas transportation in pipelines. Based on the predictions, this framework allows to fast evaluate the risk of natural gas shortages, considering the hydraulic properties of pipelines and the uncertainties in both the transportation system and the demands. Finally, a multi-objective optimization problem is developed to find the optimal price based on all the analysis results. The optimization problem is, here, solved by a genetic algorithm, named as NASG-II. To select the optimal solution from the resulting Pareto Front, a decision-making method is used, which amounts to first reducing the Pareto set by the subtractive clustering method and, then, making the final selection based on the Level 
Diagram method.

In the case study, the DSM framework is applied to a natural gas supply system, which has a complex topology structure and multiple demands, to verify its effectiveness. Considering the critical role of demand forecasting in the DSM framework, the ability of the demand forecasting model is analyzed in detail. The decision-making process is also clearly illustrated. Finally, the performance of DSM is presented from two perspectives, i.e., graphical analysis and quantification analysis, for four different pricing periods. By comparing the load profiles with and without the DSM framework, we can highlight its good performance on peak shaving and valley filling, for each pricing period. Furthermore, the quantification results show the ability of load profile smoothing, company profit improvement, peak reduction, supply reliability enhancement and customers' satisfaction. The results also indicate the pricing period should be set within 2 hours, to maintain a good flexibility.

In the future research, we intend to explore the DSM when the gas supply system is integrated with other energy systems, such as power grids, wind farms, heating systems and so on. Also, the cost of natural gas pipelines operation will be considered as an important factor for optimal price determination. Besides, this method will be applied to a real natural gas pipeline network system to further verify its effectiveness.

\section{References}

[1] R. Z. Ríos-Mercado and C. Borraz-Sánchez, "Optimization problems in natural gas transportation systems: A state-of-the-art review," Appl. Energy, vol. 147, pp. 536-555, 2015. 
Chinese natural gas supply security,” Appl. Energy, vol. 165, pp. 858-867, 2016.

[3] R. Lu, S. H. Hong, and X. Zhang, “A Dynamic pricing demand response algorithm for smart grid: Reinforcement learning approach,” Appl. Energy, vol. 220, no. February, pp. 220-230, 2018.

[4] M. A. Zehir and M. Bagriyanik, "Demand Side Management by controlling refrigerators and its effects on consumers," Energy Convers. Manag., vol. 64, pp. 238-244, Dec. 2012.

[5] A. Ghasemi, H. Shayeghi, M. Moradzadeh, and M. Nooshyar, "A novel hybrid algorithm for electricity price and load forecasting in smart grids with demand-side management," Appl. Energy, vol. 177, pp. 40-59, 2016.

[6] P. Palensky and D. Dietrich, "Demand side management: Demand response, intelligent energy systems, and smart loads," IEEE Trans. Ind. Informatics, vol. 7, no. 3, pp. 381-388, Aug. 2011.

[7] A. F. Meyabadi and M. H. Deihimi, “A review of demand-side management: Reconsidering theoretical framework," Renew. Sustain. Energy Rev., vol. 80, pp. 367-379, 2017.

[8] M. Motalleb, M. Thornton, E. Reihani, and R. Ghorbani, "Providing frequency regulation reserve services using demand response scheduling," Energy Convers. Manag., vol. 124, pp. 439-452, Sep. 2016.

[9] M. Mazidi, A. Zakariazadeh, S. Jadid, and P. Siano, "Integrated scheduling of renewable generation and demand response programs in a microgrid," Energy Convers. Manag., vol. 86, pp. 1118-1127, Oct. 2014.

[10] M. Qadrdan, M. Cheng, J. Wu, and N. Jenkins, "Benefits of demand-side response in combined gas and electricity networks," Appl. Energy, vol. 192, pp. 360-369, 2017. 
[11] J. Han and M. a. Piette, "Solutions for summer electric power shortages : Demand Response and its applications in air conditioning and refrigerating systems," 2008.

[12] P. Faria and Z. Vale, "Demand response in electrical energy supply: An optimal real time pricing approach,” Energy, vol. 36, no. 8, pp. 5374-5384, 2011.

[13] M. Zhang et al., "A systematic approach for the joint dispatch of energy and reserve incorporating demand response,” Appl. Energy, vol. 230, no. August, pp. 1279-1291, 2018.

[14] Y. Li, W. Gao, Y. Ruan, and Y. Ushifusa, "Demand response of customers in Kitakyushu smart community project to critical peak pricing of electricity,” Energy Build., vol. 168, pp. 251-260, Jun. 2018.

[15] Y. He and J. Zhang, "Real-time electricity pricing mechanism in China based on system dynamics,” Energy Convers. Manag., vol. 94, pp. 394-405, Apr. 2015.

[16] B. Mozafari, M. Bashirvand, M. Nikzad, and S. Solaymani, "A SCUC-based approach to determine time-of-use tariffs," 2012 11th Int. Conf. Environ. Electr. Eng. EEEIC 2012 - Conf. Proc., pp. 429-433, 2012.

[17] R. De Sá Ferreira, L. A. Barroso, P. R. Lino, M. M. Carvalho, and P. Valenzuela, “Time-of-use tariff design under uncertainty in price-elasticities of electricity demand: A stochastic optimization approach,” IEEE Trans. Smart Grid, vol. 4, no. 4, pp. 2285-2295, 2013.

[18] N. Nikmehr, S. Najafi-Ravadanegh, and A. Khodaei, "Probabilistic optimal scheduling of networked microgrids considering time-based demand response programs under uncertainty," Appl. Energy, vol. 198, pp. 267-279, Jul. 2017.

[19] Y. He, B. Wang, J. Wang, W. Xiong, and T. Xia, "Residential demand response behavior analysis based on Monte Carlo simulation: The case of Yinchuan in China," Energy, vol. 47, 
[20] H. A. Aalami, M. Parsa Moghaddam, and G. R. Yousefi, "Evaluation of nonlinear models for time-based rates demand response programs," Int. J. Electr. Power Energy Syst., vol. 65, pp. 282-290, Feb. 2015.

[21] N. Hajibandeh, M. Shafie-khah, G. J. Osório, J. Aghaei, and J. P. S. Catalão, “A heuristic multi-objective multi-criteria demand response planning in a system with high penetration of wind power generators," Appl. Energy, vol. 212, pp. 721-732, Feb. 2018.

[22] Y. Wang and L. Li, "Critical peak electricity pricing for sustainable manufacturing: Modeling and case studies," Appl. Energy, vol. 175, pp. 40-53, Aug. 2016.

[23] K. Herter and S. Wayland, "Residential response to critical-peak pricing of electricity: California evidence,” Energy, vol. 35, no. 4, pp. 1561-1567, Apr. 2010.

[24] H. Lin, Q. Wang, Y. Wang, Y. Liu, Q. Sun, and R. Wennersten, “The energy-saving potential of an office under different pricing mechanisms - Application of an agent-based model," Appl. Energy, vol. 202, pp. 248-258, Sep. 2017.

[25] D. Jang, J. Eom, M. G. Kim, and J. J. Rho, "Demand responses of Korean commercial and industrial businesses to critical peak pricing of electricity," J. Clean. Prod., vol. 90, pp. 275290, Mar. 2015.

[26] S. C. Park, Y. G. Jin, H. Y. Song, and Y. T. Yoon, "Designing a critical peak pricing scheme for the profit maximization objective considering price responsiveness of customers," Energy, vol. 83, pp. 521-531, Apr. 2015.

[27] J. Valenzuela, P. R. Thimmapuram, and J. Kim, "Modeling and simulation of consumer response to dynamic pricing with enabled technologies," Appl. Energy, vol. 96, pp. 122-132, 
Aug. 2012.

[28] C. Gu, X. Yan, Z. Yan, and F. Li, “Dynamic pricing for responsive demand to increase distribution network efficiency," Appl. Energy, vol. 205, pp. 236-243, Nov. 2017.

[29] H. Su, E. Zio, J. Zhang, Z. Yang, X. Li, and Z. Zhang, “A systematic hybrid method for realtime prediction of system conditions in natural gas pipeline networks," J. Nat. Gas Sci. Eng., vol. 57, no. June, pp. 31-44, 2018.

[30] I. P. Panapakidis and A. S. Dagoumas, "Day-ahead natural gas demand forecasting based on the combination of wavelet transform and ANFIS/genetic algorithm/neural network model," Energy, vol. 118, pp. 231-245, 2017.

[31] A. Demissie, W. Zhu, and C. T. Belachew, "A multi-objective optimization model for gas pipeline operations," Comput. Chem. Eng., vol. 100, pp. 94-103, 2017.

[32] H. Ahmadian Behrooz and R. B. Boozarjomehry, "Dynamic optimization of natural gas networks under customer demand uncertainties," Energy, vol. 134, pp. 968-983, 2017.

[33] İ. Durgut and M. K. Leblebicioğlu, "State estimation of transient flow in gas pipelines by a Kalman filter-based estimator,” J. Nat. Gas Sci. Eng., vol. 35, pp. 189-196, 2016.

[34] I. Güler and E. D. Übeyli, “A recurrent neural network classifier for Doppler ultrasound blood flow signals," Pattern Recognit. Lett., vol. 27, no. 13, pp. 1560-1571, 2006.

[35] E. Kuznetsova, Y. F. Li, C. Ruiz, and E. Zio, “An integrated framework of agent-based modelling and robust optimization for microgrid energy management,” Appl. Energy, vol. 129, pp. 70-88, 2014.

[36] J. Zhang, H. Zhu, C. Yang, Y. Li, and H. Wei, "Multi-objective shape optimization of helicoaxial multiphase pump impeller based on NSGA-II and ANN," Energy Convers. Manag., vol. 
52, no. 1, pp. 538-546, Jan. 2011

[37] Z. Guo, D. Chi, J. Wu, and W. Zhang, "A new wind speed forecasting strategy based on the chaotic time series modelling technique and the Apriori algorithm," Energy Convers. Manag., vol. 84, pp. 140-151, Aug. 2014.

[38] L. Zhu, M. S. Li, Q. H. Wu, and L. Jiang, "Short-term natural gas demand prediction based on support vector regression with false neighbours filtered," Energy, vol. 80, pp. 428-436, 2015.

[39] J. H. Herbert, S. Sitzer, and Y. Eades-Pryor, “A statistical evaluation of aggregate monthly industrial demand for natural gas in the U.S.A.," Energy, vol. 12, no. 12, pp. 1233-1238, Dec. 1987.

[40] Y. Chen, W. S. Chua, and T. Koch, "Forecasting day-ahead high-resolution natural-gas demand and supply in Germany," Appl. Energy, vol. 228, pp. 1091-1110, Oct. 2018.

[41] E. Čeperić, S. Žiković, and V. Čeperić, "Short-term forecasting of natural gas prices using machine learning and feature selection algorithms," Energy, vol. 140, pp. 893-900, Dec. 2017.

[42] F. Almonacid, P. J. Pérez-Higueras, E. F. Fernández, and L. Hontoria, “A methodology based on dynamic artificial neural network for short-term forecasting of the power output of a PV generator,” Energy Convers. Manag., vol. 85, pp. 389-398, Sep. 2014.

[43] A. Meng, J. Ge, H. Yin, and S. Chen, "Wind speed forecasting based on wavelet packet decomposition and artificial neural networks trained by crisscross optimization algorithm," Energy Convers. Manag., vol. 114, pp. 75-88, Apr. 2016.

[44] H. Zheng, J. Yuan, and L. Chen, "Short-Term Load Forecasting Using EMD-LSTM Neural Networks with a Xgboost Algorithm for Feature Importance Evaluation,” Energies, vol. 10, no. 8, p. 1168, 2017. 
[45] H. Liu, X. Mi, and Y. Li, "Comparison of two new intelligent wind speed forecasting approaches based on Wavelet Packet Decomposition, Complete Ensemble Empirical Mode Decomposition with Adaptive Noise and Artificial Neural Networks," Energy Convers. Manag., vol. 155, pp. 188-200, Jan. 2018.

[46] Y.-L. Hu and L. Chen, "A nonlinear hybrid wind speed forecasting model using LSTM network, hysteretic ELM and Differential Evolution algorithm," Energy Convers. Manag., vol. 173, pp. 123-142, Oct. 2018.

[47] M. Bernas and B. Płaczek, "Period-aware local modelling and data selection for time series prediction," Expert Syst. Appl., vol. 59, pp. 60-77, Oct. 2016.

[48] V. Sharma, D. Yang, W. Walsh, and T. Reindl, "Short term solar irradiance forecasting using a mixed wavelet neural network," Renew. Energy, vol. 90, pp. 481-492, 2016.

[49] X. Kong, X. Xu, Z. Yan, S. Chen, H. Yang, and D. Han, "Deep learning hybrid method for islanding detection in distributed generation,” Appl. Energy, vol. 210, no. July, pp. 776-785, 2018.

[50] A. Rahman, V. Srikumar, and A. D. Smith, "Predicting electricity consumption for commercial and residential buildings using deep recurrent neural networks," Appl. Energy, vol. 212, 2018.

[51] W. Bao, J. Yue, and Y. Rao, “A deep learning framework for financial time series using stacked autoencoders and long-short term memory," PLoS One, vol. 12, no. 7, 2017.

[52] A. Malinin, A. Ragni, K. M. Knill, and M. J. F. Gales, "Incorporating Uncertainty into Deep Learning for Spoken Language Assessment.”

[53] D. S. Kirschen, "Demand-side view of electricity markets," IEEE Trans. Power Syst., vol. 18, no. 2 , pp. 520-527, 2003. 
[54] Y. Zheng, B. M. Jenkins, K. Kornbluth, and C. Træholt, "Optimization under uncertainty of a biomass-integrated renewable energy microgrid with energy storage,” Renew. Energy, vol. 123, pp. 204-217, 2018.

[55] S. Noor, W. Yang, M. Guo, K. H. Van Dam, and X. Wang, "Energy Demand Side Management within micro-grid networks enhanced by blockchain,” Appl. Energy, vol. 228, pp. 1385-1398, 2018.

[56] D. Srinivasan, S. Rajgarhia, B. M. Radhakrishnan, A. Sharma, and H. P. Khincha, "GameTheory based dynamic pricing strategies for demand side management in smart grids," Energy, vol. 126, pp. 132-143, 2017.

[57] T. W. L. Norman, "Learning, hypothesis testing, and rational-expectations equilibrium," Games Econ. Behav., vol. 90, pp. 93-105, 2015.

[58] S. Rimkevicius et al., "Development of approach for reliability assessment of pipeline network systems," Appl. Energy, vol. 94, pp. 22-33, 2012.

[59] H. Su, E. Zio, J. Zhang, and X. Li, “A systematic framework of vulnerability analysis of a natural gas pipeline network," Reliab. Eng. Syst. Saf., vol. 175, pp. 79-91, Jul. 2018.

[60] H. Su, J. Zhang, E. Zio, N. Yang, X. Li, and Z. Zhang, “An integrated systemic method for supply reliability assessment of natural gas pipeline networks,” Appl. Energy, vol. 209, pp. 489-501, Jan. 2018.

[61] H. Su, E. Zio, J. Zhang, Z. Yang, X. Li, and Z. Zhang, “A systematic hybrid method for realtime prediction of system conditions in natural gas pipeline networks," J. Nat. Gas Sci. Eng., vol. 57, pp. 31-44, Sep. 2018.

[62] H. Su, E. Zio, J. Zhang, Z. Yang, X. Li, and Z. Zhang, “A systematic hybrid method for real- 

time prediction of system conditions in natural gas pipeline networks," J. Nat. Gas Sci. Eng., vol. 57, pp. 31-44, Sep. 2018.

[63] X. Kong, X. Xu, Z. Yan, S. Chen, H. Yang, and D. Han, "Deep learning hybrid method for islanding detection in distributed generation," Appl. Energy, vol. 210, no. April, pp. 776-785, 2018.

[64] Y. Lv, Y. Duan, W. Kang, Z. Li, and F. Y. Wang, "Traffic Flow Prediction with Big Data: A Deep Learning Approach,” IEEE Trans. Intell. Transp. Syst., vol. 16, no. 2, pp. 865-873, 2015.

[65] G. E. Hinton and R. R. Salakhutdinov, "Reducing the dimensionality of data with neural networks.," Science, vol. 313, no. 5786, pp. 504-7, Jul. 2006.

[66] S. M. Salaken, A. Khosravi, T. Nguyen, and S. Nahavandi, "Seeded transfer learning for regression problems with deep learning," Expert Syst. Appl., vol. 115, pp. 565-577, 2019.

[67] X. Fu, G. Li, and H. Wang, "Use of a second-order reliability method to estimate the failure probability of an integrated energy system,” Energy, vol. 161, pp. 425-434, Oct. 2018.

[68] E. Zio, "Some Challenges and Opportunities in Reliability Engineering," IEEE Trans. Reliab., vol. PP, no. 99, pp. 1769-1782, 2016.

[69] E. Zio, "Challenges in the vulnerability and risk analysis of critical infrastructures," Reliab. Eng. Syst. Saf., vol. 152, pp. 137-150, 2016.

[70] B. Chai, J. Chen, Z. Yang, and Y. Zhang, "Demand Response Management With Multiple Utility Companies: A Two-Level Game Approach,” IEEE Trans. Smart Grid, vol. 5, no. 2, 2014.

[71] A. Sheikhi, S. Bahrami, and A. M. Ranjbar, "An autonomous demand response program for electricity and natural gas networks in smart energy hubs," Energy, vol. 89, pp. 490-499, 2015. 
861 [72] Y. Hu, Z. Bie, T. Ding, and Y. Lin, “An NSGA-II based multi-objective optimization for

862

863

864

865

866

867

868

869

870

871

872 combined gas and electricity network expansion planning," Appl. Energy, vol. 167, pp. 280293, Apr. 2016.

[73] J. Khoury, R. Mbayed, G. Salloum, and E. Monmasson, "Predictive demand side management of a residential house under intermittent primary energy source conditions," Energy Build., vol. 112, pp. 110-120, 2016.

[74] E. Zio and R. Bazzo, "Level Diagrams analysis of Pareto Front for multiobjective system redundancy allocation,” Reliab. Eng. Syst. Saf., vol. 96, no. 5, pp. 569-580, May 2011.

[75] E. Zio and R. Bazzo, “A clustering procedure for reducing the number of representative solutions in the Pareto Front of multiobjective optimization problems," Eur. J. Oper. Res., vol. 210, no. 3, pp. 624-634, May 2011. 